\title{
Flora of Mt. Hambaek-san and its neighboring mountains
}

\author{
Doo Sub Choi, Dong Chan Son, Beom Kyun Park and Sung Chul Ko* \\ Department of Biological Science and Biotechnology, Hannam University, Daejeon 305-811, Korea \\ (Received 10 December 2014; Accepted 28 January 2015)
}

\section{함백산 일원의 식물상}

\author{
최두섭 · 손동찬 · 박범균 · 고성철* \\ 한남대학교 생명 · 나노과학대학 생명시스템과학과
}

\begin{abstract}
In order to illustrate the flora of vascular plants distributed in the area of Mt. Hambaek-san and its neighboring mountains, the authors collected vascular plants intensively on Mt. Hambaek-san (1,573 m), Mt. Geumdae-bong $(1,418 \mathrm{~m})$ and Mt. Daedeok-san $(1,307 \mathrm{~m})$ from April of 2012 to August of 2014. All of them consisted of 90 families, 310 genera, 532 species, 4 subspecies, 73 varieties and 13 forms, with a total of 622 taxa. The total number of vascular plants in the investigated area was 808 taxa $(17.55 \%$ of all vascular plants in Korea) with the addition of voucher specimens from previous investigations. Among the collected plants, endemic ones were 21 taxa, rare and endangered ones 26 taxa, naturalized ones 30 taxa, useful ones 464 taxa, and floristically specific ones of Korean floristic zones 62 taxa except for species of the first and second grade. Among the floristic zones of the Korean Peninsula, this investigated area showed the typical vegetation of a temperate middle province into which more northern elements than southern elements flowed.
\end{abstract}

Keywords: Flora, Mt. Hambaek-san, Mt. Geumdae-bong, Mt. Daedeok-san

적 요: 함백산 일원의 식물상을 파악하기 위하여 함백산 $(1,573 \mathrm{~m})$, 금대봉 $(1,418 \mathrm{~m})$, 대덕산 $(1,307 \mathrm{~m})$ 의 관속 식물을 2012년 4월부터 2014년 8월까지 조사하였다. 본 조사에서 생육이 확인된 관속식물은 90과 310속 532 종 4 아종 73 변종 13 품종의 총 622 분류군이며, 문헌상 증거표본이 있는 자료를 추가하면 총 808 분류군에 이 르고, 이는 한반도 전체 관속식물의 $17.55 \%$ 에 해당된다. 이들 중에는 한국 특산식물 21 분류군, 희귀 및 멸종 위기식물 26 분류군, 귀화식물 30 분류군, 유용자원식물은 총 464 분류군, 식물구계학적 특정식물종 62 분류군(I, II 등급 제외)이 각각 포함된다. 한편, 본 조사지역은 한반도 식물구계로 볼 때 남부식물분자보다 북부식물분 자가 더 많이 유입된 전형적인 중부아구의 식생을 보였다.

주요어: 식물상, 함백산, 금대봉, 대덕산

함백산 일원은 백두대간의 한 축을 이루는 태백산맥 중 간지점에 위치한 고산지역으로, 식물구계학적으로 일화 식물구계 온대아구계 속의 한국구 동쪽에 위치하며, 중부 아구에 속한다(Lee and Yim, 1978, 2002). 지형적으로 우리 나라에서 6 번째로 높은 함백산 $(1,573 \mathrm{~m})$ 을 중심으로 북쪽

\footnotetext{
*Author for correspondence: kscaaa@hnu.kr
}

http://www.pltaxa.or.kr

Copyright (C) 2015 the Korean Society of Plant Taxonomists
능선을 따라 은대봉 $(1,442 \mathrm{~m})$, 금대봉 $(1,418 \mathrm{~m})$, 대덕산 $(1,307 \mathrm{~m})$ 이 연결되며(Fig. 1), 함백산 일원의 중앙부에 위 치한 금대봉을 중심으로 북서쪽에는 노목산 $(1,145 \mathrm{~m})$, 서 쪽에는 백운산 $(1,426 \mathrm{~m})$, 동쪽에는 매봉산 $(1,303 \mathrm{~m})$ 과 인접 하고, 남쪽에는 태백산도립공원이 자리잡고 있다. 태백산 맥에 속하여 대체적으로 능선의 동사면은 높고 서사면은 낮아 완만한 경사를 이루는 경동지형의 특색을 가지며, $1,000 \mathrm{~m}$ 이상의 산지들이 수계를 이루므로 비교적 수질이 좋고, 식생 피복이 양호한 편이다(Kim and Park, 2009).

기후는 내륙산간지대의 특징인 대륙성기후로 기온의 
연교차와 일교차가 심하고, 여름은 서늘하며, 겨울은 몹시 춥다. 최근 5 년간 연평균 기온은 $10.0^{\circ} \mathrm{C}$ 내외, 연평균 강수량 은 1,274.2 mm 정도이다(Korean Meterological Administration, $2008,2009,2010,2011,2012)$. 토양은 온대중부의 갈색산 림토지역에 속한다(Kang, 1971).

함백산 일대는 국내 유수의 탄전지대이기도 하며, 산업 선인 태백선 철도가 북동사면을 지난다. 함백산의 북서사 면에는 선덕여왕 5 년에 자장율사가 창건한 정암사가 있 고, 그 인근의 계곡부에서는 천연기념물 제 73 호인 열목 어서식지가 있으며(Jeongseon county, 2012), 만항재 $(1,330 \mathrm{~m})$ 부근에는 국내 최대 규모의 야생화 군락지가 조 성되어 있다. 또한, 금대봉과 대덕산 지역에는 강원도 지 방기념물 제 39 호인 용연굴과 한강의 발원지로 알려진 검 룡소가 있으며(Taebaek city, 2012), 이 일대는 생태계 보전 가치가 높아 1993년부터 이 일대 면적 $4.20 \mathrm{~km}^{2}$ 를 자연생태 계보전지역으로 지정하여 보호하고 있다(Kim et al., 2004). 특히, 복주머니란(Cypripedium macranthon Sw.), 한계령풀 (Leontice microrrhyncha S. Moore), 대성쓴풀[Anagallidium dichotomum (L.) Grisb.] 등 학술적으로 중요한 희귀식물의 생육이 다수 보고되고 있는 지역이기도 하다.

함백산 일원의 식물상에 관한 선행조사로 함백산은 Lee and Oh (1999)에 의한 함백산(정선 · 태백)과 인근산지의 식 물상 77 과 248속 340 종 1 아종 72 변종 12품종의 총 425 분류 군이 보고된 이후, $\operatorname{Kim}$ (2001)에 의해 79과 261속 365종 2아 종 75변종 11품종의 총 453분류군, Oh et al. (2009)에 의해 36 과 95 속 99 종 1 아종 14 변종 3품종의 총 117 분류군, Kim and Park (2012)에 의해 64과 184속 203종 3아종 48변종 11품 종의 총 265 분류군이 각각 조사된 바 있으며, 금대봉은 $\mathrm{Oh}$ et al. (2009)이 76과 230속 316종 1아종 40변종 4품종의 총 361 분류군, 대덕산은 Seo et al. (2006)이 81과 266속 358종 1 아종 69 변종 14 품종의 총 442 분류군을 보고한 바 있다. 그 외 조사로는 Park et al. (2002)의 함백산 지역 계곡부의 사면 방향과 해발고도에 따른 산림구조가 연구된 바 있다.

그러나, 이들 선행연구의 식물목록을 검토해보면 왕둥 굴레[Polygonatum robustum (Korsch.) Nakai], 민등골나물 (Eupatorium chinense var. angustatum H. Hara), 나도여로 [Zygadenus sibiricus (L.) A. Gray] 등 분류학적 실체가 불분 명하고, 분포지가 의심스러운 분류군이 다수 포함되어 있 으며, 식물종의 생육지 정보 및 증거표본에 대한 언급이 Lee and Oh (1999)와 Oh et al. (2009) 외에는 제시된 바 없기 때문에 식물상의 비교 연구를 수행하는데 어려움이 있다. 또한, 대덕산 서사면과 북사면, 금대봉의 남동사면 일대 에는 고랭지밭이 넓게 형성되어 있으며, 경작지의 면적도 해마다 늘어나고 있어, 자연림의 훼손이 매우 심각한 실 정이다. 또한, 함백산은 접근이 용이하고, 동사면 일대에 는 골프장과 스키장이 조성되어 시간이 경과함에 따라 삼 림의 피해가 증가될 것으로 예상된다.

따라서, 본 연구는 우선적으로 함백산 일원에 대한 현지
조사를 통해 증거표본을 수집하여 그에 기초한 정확한 관 속식물상을 확임함은 물론, 본 지역의 식물지리학적 특이 성과 중요성을 파악하는 동시에 특산식물과 희귀식물 등 주요 분류군의 분포를 파악하여 함백산 일원의 종다양성 보존을 위한 기초자료를 제공하고자 수행되었다.

\section{재료 및 방법}

\section{조사일정}

조사지역인 함백산 일원의 관속식물상을 조사하기 위 하여 2012년 4월부터 2014년까지 8월까지 총 32회에 걸쳐 현지조사를 실시하였으며, 조사일정과 경로는 Table 1, Fig. 1과 같다.

\section{표본 수집, 제작 및 동정}

함백산 일원의 정확한 관속식물상 파악을 위해 꽃, 열매, 포자 등의 생식기관이 있는 개체만을 채집하였다. 채집된 식물들은 압착 - 건조하여 표본으로 제작한 후 각 표본에 는 채집번호와 좌표를 부여하였고, 동정은 Lee (1980, 2003), Lee $(1996,2006)$ 등의 도감류와 Park (2007)의 식물 지 등을 이용하였으며, 확증표본을 바탕으로 확인된 종만 을 기재하였다. 각 표본에는 채집번호와 좌표를 부여하였 으며, 증거표본은 한남대학교 자연사박물관(HNHM), 국 립생물자원관(KB), 국립수목원(KH)에 각각 보관하였다.

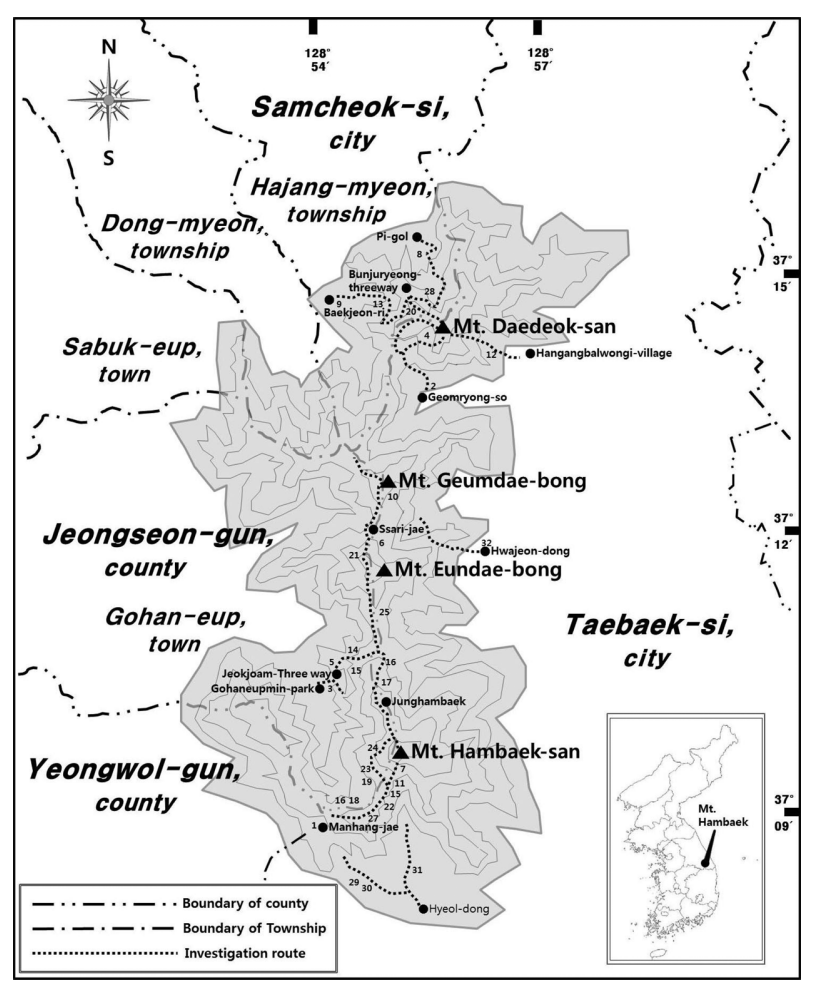

Fig. 1. Map of investigation area, Gangwon-do province, and routes. 
Table 1. Dates and routes of the investigations.

\begin{tabular}{|c|c|c|}
\hline No. & Date & Investigation routes \\
\hline 1 & 18 Apr. 2012 & Manhang-jae (uphill) $\rightarrow$ Alt. 1,338m, southwest slope of Hambaek-san (Mt.) $\rightarrow$ Forest road $\rightarrow$ Manhang-jae (uphill). \\
\hline 2 & 19 Apr. 2012 & $\begin{aligned} \text { Geomryong-so (spring) } & \rightarrow \text { Bunjujae-gol (valley) } \rightarrow \text { Jeongjinwoebak-gol (valley) } \rightarrow \text { Alt. 1,323 m, southwest slope } \\
\text { of Daedeok-san (Mt.) } & \rightarrow \text { Bunjuryeong (valley) } \rightarrow \text { Geomryong-so (spring). }\end{aligned}$ \\
\hline 3 & 24 May 2012 & $\begin{array}{l}\text { Gohaneubmincheyuk-gongwon (park) } \rightarrow \text { Jeokjoam-samgeori (three-way) } \rightarrow \text { Jajaknamu-saemteo (spring) } \rightarrow \\
\text { Junghambaek (Mt.) } \rightarrow \text { Summit of Hambaek-san (Mt.) } \rightarrow \text { Manhang-jae (uphill). }\end{array}$ \\
\hline 4 & 25 May 2012 & $\begin{array}{l}\text { Geomryong-so (spring) } \rightarrow \text { Bunjujae-gol (valley) } \rightarrow \text { Jeongjinwoebak-gol (valley) } \rightarrow \text { Summit of Daedeok-san (Mt.) } \\
\rightarrow \text { Hongchimi-gol (valley) } \rightarrow \text { Hangang-balwongi(village). }\end{array}$ \\
\hline 5 & 03 Jul. 2012 & $\begin{array}{l}\text { Gohaneubmincheyuk-gongwon (park) } \rightarrow \text { Jeokjoam-samgeori (three-way) } \rightarrow \text { Jajaknamu-saemteo (spring) } \\
\rightarrow \text { Alt. } 1,300 \text { m, northwest slope of Hambaek-san }(M t .) \rightarrow \text { Gohaneubmincheyuk-gongwon (park). }\end{array}$ \\
\hline 6 & 04 Jul. 2012 & $\begin{array}{l}\text { Ssari-jae (uphill) } \rightarrow \text { Heliport } \rightarrow \text { Alt. 1,339 m, northern slope of Eundae-bong (Mt.) } \rightarrow \text { Ssari-jae (uphill). } \\
\text { Ssari-jae (uphill) } \rightarrow \text { Summit of Geumdae-bong (Mt.) } \rightarrow \text { Gomoknamu-saemteo (spring) } \rightarrow \text { Ssari-jae (uphill). }\end{array}$ \\
\hline 7 & 05 Jul. 2012 & Forest ranger station $\rightarrow$ Heliport $\rightarrow$ Summit of Hambaek-san (Mt.) $\rightarrow$ Forest ranger station. \\
\hline 8 & 18 Jul. 2012 & $\begin{array}{l}\text { Pi-gol (valley) } \rightarrow \text { Bunjuryeong-samgeori (three-way) } \rightarrow \text { Bunju-ryeong (valley) } \rightarrow \text { Alt. 1,170 m northwest slope of } \\
\text { Daedeok-san }(\text { Mt.) } \rightarrow \text { Pi-gol (valley). }\end{array}$ \\
\hline 9 & 14 Aug. 2012 & $\begin{array}{l}\text { Baekjeon-ri } \rightarrow \text { Jeol-gol (valley) } \rightarrow \text { Bunjuryeong-samgeori (three-way) } \rightarrow \text { Alt. 1,166 m, northwest slope of } \\
\text { Daedeok-san } \rightarrow \text { Baekjeon-ri. }\end{array}$ \\
\hline 10 & 15 Aug. 2012 & $\begin{array}{l}\text { Ssari-jae (uphill) } \rightarrow \text { Alt. 1,348 m, northern slope of Geumdae-bong (Mt.) } \rightarrow \text { Gomoknamu-saemteo (spring) } \\
\rightarrow \text { Ssari-jae (uphill). }\end{array}$ \\
\hline 11 & 19 Sep. 2012 & Forest ranger station $\rightarrow$ Summit of Hambaek-san (Mt.) $\rightarrow$ Heliport $\rightarrow$ Forest ranger station $\rightarrow$ Manhang-jae (uphill). \\
\hline 12 & 20 Sep. 2012 & $\begin{array}{l}\text { Geomryong-so (spring) } \rightarrow \text { Bunjujae-gol (valley) } \rightarrow \text { Jeongjinwoebak-gol (valley) } \rightarrow \text { Summit of Daedeok-san (Mt.) } \\
\rightarrow \text { Hongchimi-gol (valley) } \rightarrow \text { Geomryong-so (spring). }\end{array}$ \\
\hline 13 & 24 Oct. 2012 & $\begin{array}{l}\text { Jeol-gol (valley) } \rightarrow \text { Bunjuryeong-samgeori (three-way) } \rightarrow \text { Alt. 1,108 m, northwest slope of Daedeok-san (Mt.) } \\
\rightarrow \text { Yangmae-gol (valley) } \rightarrow \text { Jeol-gol (valley). }\end{array}$ \\
\hline 14 & 25 Oct. 2012 & $\begin{array}{l}\text { Gohaneubmincheyuk-gongwon (park) } \rightarrow \text { Jeokjoam-samgeori (three-way) } \rightarrow \text { Alt. 1,166 m, northwest slope of } \\
\text { Hambaek-san (Mt.) } \rightarrow \text { Gohaneubmincheyuk-gongwon (park). }\end{array}$ \\
\hline & & $\begin{array}{l}\text { Manhang-jae (uphill) } \rightarrow \text { Hambaeksan-gongwon (park) } \rightarrow \text { Alt. 1,287 m, southwest slope of Hambaek-san (Mt.) } \\
\rightarrow \text { Manhang-jae (uphill). }\end{array}$ \\
\hline 15 & 11 Apr. 2013 & $\begin{array}{l}\text { Gohaneubmincheyuk-gongwon (park) } \rightarrow \text { Jeokjoam-samgeori (three-way) } \rightarrow \text { Alt. } 917 \mathrm{~m} \text {, northwest slope of } \\
\text { Hambaek-san (Mt.) } \rightarrow \text { Gohaneubmincheyuk-gongwon (park). }\end{array}$ \\
\hline & & Manhang-jae (uphill) $\rightarrow$ Jangsan-condo (hotel). \\
\hline 16 & 12 Apr. 2013 & $\begin{array}{l}\text { Manhang-jae (uphill) } \rightarrow \text { Hambaeksan-gongwon (park) } \rightarrow \text { Alt. 1,369 m, southwest slope of Hambaek-san (Mt.) } \\
\rightarrow \text { Manhang-jae (uphill). }\end{array}$ \\
\hline & & Jeongam-sa (temple) $\rightarrow$ Alt. 801 m, northwest slope of Hambaek-san (Mt.) $\rightarrow$ Jeongam-sa (temple). \\
\hline 17 & 01 May 2013 & $\begin{array}{l}\text { Gohaneubmincheyuk-gongwon (park) } \rightarrow \text { Jeokjoam-samgeori (three-way) } \rightarrow \text { Jajaknamu-saemteo (spring) } \\
\rightarrow \text { Junghambaek (Mt.) } \rightarrow \text { Alt. 1,467 m, western slope of Hambaek-san }(\text { Mt.) } \rightarrow \text { Heliport } \rightarrow \text { Forest ranger station. }\end{array}$ \\
\hline 18 & 02 May 2013 & Forest ranger station $\rightarrow$ Manhang-jae (uphill). \\
\hline 19 & 24 Jun. 2013 & $\begin{array}{l}\text { Manhang-jae (uphill) } \rightarrow \text { Forest ranger station } \rightarrow \text { Alt. 1,321 m, southwest slope of Hambaek-san (Mt.) } \\
\rightarrow \text { Manhang-jae (uphill). }\end{array}$ \\
\hline 20 & 10 Jul. 2013 & $\begin{array}{l}\text { Baekjeon-ri } \rightarrow \text { Jeol-gol (valley) } \rightarrow \text { Bunjuryeong-samgeori (three-way) } \rightarrow \text { Alt. 1,176 m, northwest slope of } \\
\text { Daedeok-san } \rightarrow \text { Baekjeon-ri. }\end{array}$ \\
\hline 21 & 11 Jul. 2013 & Ssari-jae (uphill) $\rightarrow$ Summit of Eundae-bong (Mt.) $\rightarrow$ Ssari-jae (uphill). \\
\hline 22 & 28 Aug. 2013 & $\begin{array}{l}\text { Forest ranger station } \rightarrow \text { Summit of Hambaek-san (Mt.) } \rightarrow \text { Junghambaek (Mt.) } \rightarrow \text { Jajaknamu-saemteo (spring) } \\
\rightarrow \text { Jeokjoam- samgeori (three-way) } \rightarrow \text { Gohaneubmincheyuk-gongwon (park). }\end{array}$ \\
\hline 23 & 29 Aug. 2013 & Manhang-jae (uphill) $\rightarrow$ Alt. 1,162 m, southwest slope of Hambaek-san (Mt.) $\rightarrow$ Manhang-jae (uphill). \\
\hline 24 & 03 Oct. 2013 & Manhang-jae (uphill) $\rightarrow$ Forest ranger station $\rightarrow$ Summit of Hambaek-san (Mt.) $\rightarrow$ Manhang-jae (uphill). \\
\hline
\end{tabular}


Table 1. Continued.

\begin{tabular}{|c|c|c|}
\hline No. & Date & Investigation routes \\
\hline 25 & 24 Apr. 2014 & Ssari-jae (uphill) $\rightarrow$ Summit of Eundae-bong (Mt.) $\rightarrow$ Ssari-jae (uphill). \\
\hline 26 & 25 Apr. 2014 & $\begin{array}{l}\text { Gohaneubmincheyuk-gongwon (park) } \rightarrow \text { Jeokjoam-samgeori (three-way) } \rightarrow \text { Jajaknamu-saemteo (spring) } \\
\rightarrow \text { Alt. } 1,308 \text { m, northwest slope of Hambaek-san }(\text { Mt.) } \rightarrow \text { Gohaneubmincheyuk-gongwon (park). }\end{array}$ \\
\hline 27 & 22 May 2014 & $\begin{array}{l}\text { Forest ranger station } \rightarrow \text { Alt. 1,465 m, southwest slope of Hambaek-san (Mt.) } \rightarrow \text { Junghambaek (Mt.) } \\
\rightarrow \text { Forest ranger station. }\end{array}$ \\
\hline & & Hwangji-dong (village) $\rightarrow$ Alt. 1,366 m, east slope of Hambaek-san (Mt.) $\rightarrow$ Hwangii-dong (village). \\
\hline 28 & 23 May 2014 & $\begin{array}{l}\text { Jeol-gol (valley) } \rightarrow \text { Bunjuryeong-samgeori (three-way) } \rightarrow \text { Alt. 1,090 m, northwest slope of Daedeok-san (Mt.) } \\
\rightarrow \text { Jeol-gol (valley). }\end{array}$ \\
\hline 29 & 05 Jun. 2014 & Hyeol-dong (village) $\rightarrow$ Mak-gol (valley) $\rightarrow$ Alt. 999 m, southwest slope of Hambaek-san (Mt.) $\rightarrow$ Hyeol-dong (village). \\
\hline 30 & 04 Jul. 2014 & Hyeol-dong (village) $\rightarrow$ Mak-gol (valley) $\rightarrow$ Alt. 1,091 m, southwest slope of Hambaek-san (Mt.) $\rightarrow$ Hyeol-dong (village). \\
\hline 31 & 05 Jul. 2014 & Hyeol-dong (village) $\rightarrow$ Sanae-gol (valley) $\rightarrow$ Alt. 1,189 m, southern slope of Hambaek-san (Mt.) $\rightarrow$ Hyeol-dong (village). \\
\hline 32 & 07 Aug. 2014 & Hwajeon-dong (village) $\rightarrow$ Alt. 990 m, southeast slope of Guemdae-bong (Mt.) $\rightarrow$ Hwajeon-dong (village). \\
\hline
\end{tabular}

\section{소산식물 목록작성}

관속식물의 목록 작성은 Engler의 분류체계(Melchior, 1964)에 따라 작성되었고, 과 내에서는 속명과 종명을 알 파벳순으로 정리하였다. 학명의 사용은 A Synonymic List of Vascular Plants in Korea (Korea National Arboretum and The Plant Taxonomic Society of Korea, 2007)에 따랐다. 작성 된 소산식물목록(Appendix 1)을 기초로 한국특산식물은 Oh et al. (2005)에 의하였고, 식물구계학적특정식물종과 멸종위기종은 Ministry of Environment and National Institute of Environmental Research (2006)에 따라 파악하였으며, 희 귀식물은 Rare Plants Data Book in Korea (Korea Forest Service and Korea National Arboretum, 2008)를, 귀화식물은 Park (2009)과 Lee et al. (2011)을 따라 작성하였다. 또한, 식 물의 유용도 분석은 Lee (1976)에 준하였고, 소산식물목록 (Appendix 2)에 표기하였다.

본 논문에 수록된 식물목록(Appendix 1)에는 과명, 학명, 국명과 채집번호만이 포함되어 있으며, 구체적인 조사지 별 분포여부, 용도 등이 포함된 종합식물목록(Appendix 2) 은 교신저자(kscaaa@hnu.kr) 또는 한국식물분류학회 홈페 이지(http://www.pltaxa.or.kr)로부터 확인할 수 있다.

\section{결과 및 고찰}

\section{식물상}

1) 증거표본에 기초한 함백산 일원의 관속식물상

본 연구에서 밝혀진 함백산 일원의 관속식물은 90 과 310 속 532종 4아종 73변종 13품종의 총 622분류군이며, 이 는 한반도 관속식물 4,071 (Lee, 1996)의 약 $15.2 \%$ 에 해당되 고, 강원도 관속식물 1,465 분류군(Oh et al., 2009)의 약 $42.3 \%$ 에 이른다. 한편, 조사된 분류군 수가 가장 많았던 지역은 함백산(543분류군), 대덕산(311분류군), 금대봉 (106분류군)의 순서이고, 이는 산세의 규모와 그에 따른
조사 횟수의 차이 때문으로 생각된다. 한편, 증거표본이 제시된 이전 조사 결과(Lee and Oh, 1999; Oh et al., 2009)와 통합하면 총 808 분류군에 이르며, 이는 한반도 전체식물 4,602분류군(Lee, 1976)에 대비하여 $17.55 \%$ 로서 매우 높은 수치이다(Table 2).

조사된 함백산 일원의 관속식물은 622 분류군으로 인근 산지인 덕항산(1,077 m) 590분류군(Kim et al., 2005), 백운 산 $(883 \mathrm{~m})$ 604분류군(Song, 2000)보다 종다양성이 높은 것 으로 확인되었고, 도립공원인 태백산 $(1,567 \mathrm{~m})$ 의 633 분류 군(Lee, 1987), 청옥산 $(1,404 \mathrm{~m})$ 과 두타산 $(1,353 \mathrm{~m})$ 686분류 군(Cho et al., 1999)과 유사한 수치를 보였다. 한편, 이전 식 물상 조사에서 금대봉 361분류군(Oh et al., 2009), 대덕산 442분류군(Seo et al., 2006)에 비해서는 종다양성이 다소 낮은 것으로 조사되었다. 이는 금대봉과 대덕산이 자연생 태보전지역으로 지정되어 증거표본수집이 가능하지 않 았기 때문이다. 그렇지만 추후 조사 경로와 일정을 달리 하여 보완조사를 수행한다면, 함백산 일원의 분류군 수는 그 산세로 볼 때 더욱 증가될 것이라 생각된다.

조사지역은 중부아구 동쪽에 자리한 대표적인 온대식 생을 보이며(Seo et al., 2006), 전체적으로 신갈나무(Quercus mongolica Fisch. ex Ledeb.) 군락이 능선부와 계곡부 등에 넓 게 분포하고, 저지대에는 일본잎갈나무[Larix kaempferi (Lamb.) Carriére]가 넓게 식재되어 있으며, 고지대로 갈수록 신갈나무의 출현 비율이 비교적 높아진다. 특히, 함백산과 금대봉의 서사면 정암사 계곡부는 상당기간 출입이 금지 된 지역으로 생태적 지위와 안정성이 비교적 높기 때문에 동사면에 비해 식생상태가 양호하며(Park et al., 2002), 피나 무(Tilia amurensis Rupr.), 자작나무[Betula platyphylla var. japonica (Miq.) Hara], 당단풍나무[Acer pseudosieboldianum (Pax) Kom.], 생강나무(Lindera obtusiloba Blume), 팥배나무 [Sorbus alnifolia (Siebold \& Zucc.) K. Koch] 등이 자주 출현 하고, 중함백 인근에는 너도바람꽃(Eranthis stellata Maxim.), 
홀아비바람꽃(Anemone koraiensis Nakai), 기생꽃[Trientalis europaea var. arctica (Fisch.) Ledeb.] 등의 생육이 확인되었 다. 대덕산의 경우 북사면은 상당수의 일본잎갈나무가 식 재되어 있고, 가래나무(Juglans mandshurica Maxim.), 까치 박달(Carpinus cordata Blume), 고로쇠나무 [Acer pictum subsp. mono (Maxim.) Ohashi] 등이 자주 출현하였다. 또한, 남사면 에는 박달나무(Betula schmidtii Regel), 물푸레나무(Fraxinus rhynchophylla Hance), 복장나무(Acer mandshuricum Maxim.) 등이 분포하고 있으며, 정상부에는 노랑무늬붓꽃(Iris odaesanensis Y.N. Lee), 검종덩굴(Clematis fusca Turcz.), 금 강제비꽃(Viola diamantiaca Nakai) 군락 등의 희귀식물 군 락들이 다수 분포하고 있다.

증거표본에 기초하여 조사된 관속식물은 분류체계에 따라 구분하면, 양치식물은 8 과 12 속 20 종의 20 분류군 (3.2\%), 나자식물은 3 과 5 속 5 종의 5 분류군 $(0.8 \%)$, 피자식물 의 쌍자엽식물은 70 과 241 속 424 종 4 아종 64 변종 10 품종의
502 분류군 $(80.7 \%)$, 단자엽식물은 9과 52속 83종 9변종 3품 종의 95분류군(15.3\%)으로 각각 구성되어 있다(Table 3).

식물상 종구성의 주요 10 개과는 국화과 식물이 74 분류 군(11.89\%)으로 가장 많았고, 범의귀과, 꿀풀과, 사초과 식 물이 20 분류군 $(3.21 \%)$ 으로 가장 적었으며, 이들 10 개 과가 차지하는 비율은 $51.24 \%$ 로 전체 식물상의 약 $1 / 2$ 에 해당 된다(Table 4).

2) 본 연구를 통해 추가 확인된 분류군

본 연구와 관련된 증거표본이 제시되어 있는 기존 연구 자료(Lee and Oh, 1999; Oh et al., 2009)와 비교 검토를 한 결 과, 본 연구를 통해 새로이 분포가 확인된 분류군은 석송 (Lycopodium clavatum L.), 참개별꽃 [Pseudostellaria coreana (Nakai) Ohwi], 너도바람꽃, 흰괭이눈[Chrysosplenium pilosum var. fulvum (N. Terracc.) Hara], 붉은터리풀[Filipendula koreana (Nakai) Nakai], 털둥근이질풀(Geranium koreanum

Table 2. Comparison of floras in this investigation with those in previous ones*.

\begin{tabular}{lccccccc}
\hline \hline Mountains & Family & Genus & Species & Subsp. & Var. & For. & Total taxa \\
\hline Hambaek-san & 84 & 280 & 462 & 4 & 67 & 10 & 543 \\
Geumdae-bong & 35 & 89 & 90 & 1 & 15 & - & 106 \\
Daedeok-san & 70 & 198 & 268 & 3 & 35 & 5 & 311 \\
\hline Subtotal taxa & 90 & 310 & 532 & 4 & 73 & 13 & 622 \\
\hline Hambaek-san* & 80 & 249 & 350 & 2 & 64 & 8 & 424 \\
Hambaek-san** & 36 & 95 & 99 & 1 & 14 & 3 & 117 \\
Geumdae-bong** & 76 & 230 & 316 & 1 & 40 & 4 & 361 \\
\hline Total taxa & 95 & 354 & 679 & 5 & 106 & 18 & 808 \\
\hline
\end{tabular}

*Lee and Oh (1999); **Oh et al. (2009).

Table 3. Summary on the flora of Mt. Hambaek-san and its neighboring mountains.

\begin{tabular}{ccccccccc}
\hline \hline Taxa & Fam. & Gen. & Sp. & Subsp. & Var. & For. & Total & Ratio (\%) \\
\hline Pteridophyta & 8 & 12 & 20 & - & - & - & 20 & 3.2 \\
Gymnospermae & 3 & 5 & 5 & - & - & - & 5 & 0.8 \\
Angiospermae & & & & & & & & \\
Dicotyledons & 70 & 241 & 424 & 4 & 64 & 10 & 502 & 80.7 \\
Monocotyledons & 9 & 52 & 83 & - & 9 & 3 & 95 & 15.3 \\
\hline Total & 90 & 310 & 532 & 4 & 73 & 13 & 622 & 100 \\
\hline
\end{tabular}

Table 4. The list of major families in Mt. Hambaek-san and its neighboring mountains.

\begin{tabular}{lcclcc}
\hline \hline \multicolumn{1}{c}{ Family } & Taxa (No.) & Ratio (\%) & Family & Taxa (No.) & Ratio (\%) \\
\hline Compositae & 74 & 11.89 & Leguminosae & 27 & 4.34 \\
Rosaceae & 42 & 6.75 & Gramineae & 25 & 4.01 \\
Ranunculaceae & 32 & 5.14 & Cyperaceae & 20 & 3.21 \\
Liliaceae & 31 & 4.98 & Saxifragaceae & 20 & 3.21 \\
Umbelliferae & 28 & 4.50 & Labiatae & 20 & 3.21 \\
\hline
\end{tabular}


var. hirsutum Nakai), 독미나리(Cicuta virosa L.), 백부자 (Aconitum koreanum R. Raymund) 등을 포함한 총 253분류 군이며, 이들은 소산식물목록(Appendix 1)의 일련번호 앞 에 “`”로 표시하였다. 한편, 골고사리(Asplenium scolopendrium L.), 만삼[Codonopsis pilosula (Franch.) Nannf.], 개 버무리(Clematis serratifolia Rehder), 도깨비부채 (Rodgersia podophylla A. Gray) 등은 본 조사지역 내에 생 육이 확인되었으나, 포자, 꽃, 열매 등의 생식기관이 없어 채집되지 않았고, 이에 따라 전체 식물상 목록에서는 제 외되었다.

\section{특기할 만한 식물}

1)한국특산식물

본 조사지역에서 생육이 확인된 한국특산식물은 참개 별꽃, 진범(Aconitum pseudolaeve Nakai), 요강나물[Clematis fusca var. coreana (H. Lév. \& Vaniot) Nakai], 금꿩의다리 [Thalictrum rochebrunianum var. grandisepalum (H. Lév.) Nakai], 고려엉겅퀴[Cirsium setidens (Dunn) Nakai] 등 총 12 과 21 분류군이다. 이는 함백산 일원의 관속식물 622 분류 군의 $3.38 \%$ 를 차지하며, 증거표본이 있는 기존 연구자료 (Lee and Oh, 1999; Oh et al., 2009)를 포함하면 총 28분류군

Table 5. Korean endemic plants found in the present and the previous investigations.

\begin{tabular}{|c|c|c|c|c|c|c|c|}
\hline \multirow{2}{*}{ No. } & \multirow{2}{*}{ Scientific name } & \multicolumn{6}{|c|}{ Locality } \\
\hline & & 1 & 2 & 3 & 4 & 5 & 6 \\
\hline 1 & Salix koriyanagi Kimura 키버들 & $\bigcirc$ & & & $\bigcirc$ & & \\
\hline 2 & Pseudostellaria coreana (Nakai) Ohwi 참개별꽃 & $\bigcirc$ & & & & & \\
\hline 3 & Aconitum pseudolaeve Nakai 진범 & $\bigcirc$ & $\bigcirc$ & & $\bigcirc$ & $\bigcirc$ & $\bigcirc$ \\
\hline 4 & Anemone koraiensis Nakai 홀아비바람꽃 & $\bigcirc$ & & & $\bigcirc$ & & O \\
\hline 5 & Clematis fusca var. coreana (H. Lév. \& Vaniot) Nakai 요강나물 & $\bigcirc$ & & & $\bigcirc$ & & O \\
\hline 6 & Clematis trichotoma Nakai 할미밀 망 & $\bigcirc$ & & & $\bigcirc$ & & O \\
\hline 7 & Thalictrum rochebrunianum var. grandisepalum (H. Lév.) Nakai 금뀡의다리 & & & $\bigcirc$ & & & \\
\hline 8 & Berberis koreana Palib. 매자나무* & & & & $\bigcirc$ & & \\
\hline 9 & Corydalis grandicalyx B.U. Oh \& Y.S. Kim 갈퀴현호색 & $\bigcirc$ & & & & & $\bigcirc$ \\
\hline 10 & Corydalis maculata B.U. Oh \& Y.S. Kim 점현호색* & & & & & & $\bigcirc$ \\
\hline 11 & Cardamine koreana (Nakai) Nakai 참고추냉이* & & & & $\bigcirc$ & & \\
\hline 12 & Sedum latiovalifolium Y.N. Lee 태백기린초 & $\bigcirc$ & $\bigcirc$ & $\bigcirc$ & & & \\
\hline 13 & Chrysosplenium pilosum var. fulvum (N. Terracc.) Hara 흰갱이눈 & $\bigcirc$ & & & & & O \\
\hline 14 & Philadelphus schrenkii Rupr. 고광나무* & & & & $\bigcirc$ & & O \\
\hline 15 & Vicia chosenensis Ohwi 노랑갈퀴 & $\bigcirc$ & & $\bigcirc$ & & & $\bigcirc$ \\
\hline 16 & Geranium knuthii Nakai 큰세잎쥐손이 & $\bigcirc$ & & & & & \\
\hline 17 & Geranium taebaek S.J. Park \& Y.S. Kim 태백이질풀 & $\bigcirc$ & & & & & \\
\hline 18 & Angelica cartilagino-marginata var. distans (Nakai) Kitag. 흰바디나물* & & & & $\bigcirc$ & & \\
\hline 19 & Forsythia koreana (Rehder) Nakai 개나리* & & & & $\bigcirc$ & & $\bigcirc$ \\
\hline 20 & Salvia chanryoenica Nakai 참배암차즈기 & $\bigcirc$ & & & & & \\
\hline 21 & Pedicularis resupinata var. umbrosa Kom. ex Nakai 그늘송이풀* & & & & $\bigcirc$ & & \\
\hline 22 & Lonicera subsessilis Rehder 청괴불나무 & $\bigcirc$ & & & & & \\
\hline 23 & Weigela subsessilis (Nakai) L.H. Bailey 병꽃나무 & $\bigcirc$ & $\bigcirc$ & $\bigcirc$ & & & \\
\hline 24 & Aster koraiensis Nakai 벌개미취 & $\bigcirc$ & & & $\bigcirc$ & & \\
\hline 25 & Cirsium setidens (Dunn) Nakai 고려엉겅퀴 & $\bigcirc$ & $\bigcirc$ & $\bigcirc$ & $\bigcirc$ & $\bigcirc$ & $\bigcirc$ \\
\hline 26 & Crepidiastrum koidzumianum (Kitam.) Pak \& Kawano 지리고들빼기 & $\bigcirc$ & & & & & \\
\hline 27 & Saussurea macrolepis (Nakai) Kitam. 각시서덜취 & $\bigcirc$ & & $\bigcirc$ & & $\bigcirc$ & $\bigcirc$ \\
\hline 28 & Carex okamotoi Ohwi 지리대사초 & 0 & & & 0 & & \\
\hline
\end{tabular}

1, Mt. Hambaek-san; 2, Mt. Geumdae-bong; 3, Mt. Daedeok-san; 4, Mt. Hambaek-san(Lee and Oh, 1999); 5, Mt. Hambaek-san(Oh et al., 2009); 6, Mt. Geumdae-bong(Oh et al., 2009); *, Previous investigations. 
에 이른다(Table 5). 이 중 참개별꽃은 함백산 남사면 혈동 사내골 부근에서 소군락 단위로 생육하고 있었고, 태백이 질풀(Geranium taebaek S.J. Park \& Y.S. Kim)은 둥근이질풀 (Geranium koreanum Kom.)에 비해 꽃잎의 끝이 2개로 갈 라지는 특징으로 쉅게 구분되는 분류군으로 만항재 일대 에서 비교적 흔히 관찰되었으며, 큰세잎쥐손이(Geranium knuthii Nakai)는 만항재 인근의 산불감시초소에서 함백산 정상에 이르는 구간의 초입에 소수 개체가 발견되었다.
2) 희귀식물

조사지역의 희귀식물(Korea Forest Service and Korea National Arboretum, 2008)은 16과 26분류군이 확인되었다 (Table 6). 등급별로 구분해 보면 야생멸종 $(\mathrm{EW})$ 등급의 종은 발견되지 않았고, 멸종위기종(CR)은 백부자, 독미나리, 대 성쓴풀의 총 3 분류군이 확인되었다. 특히, 백부자는 최근 약재로서 효능이 알려지면서 개체수가 급격히 줄어들고 있는 종으로 금대봉의 싸리재 부근에서 생육이 확인되었

Table 6. Rare plants found in the present and the previous investigations.

\begin{tabular}{|c|c|c|c|c|c|c|c|c|}
\hline \multirow{2}{*}{ No. } & \multirow{2}{*}{ Scientific name } & \multirow{2}{*}{ Grade } & \multicolumn{6}{|c|}{ Locality } \\
\hline & & & 1 & 2 & 3 & 4 & 5 & 6 \\
\hline 1 & Aconitum koreanum R. Raymund 백부자 & $\mathrm{CR}$ & & O & & & & \\
\hline 2 & Cicuta virosa $\mathrm{L}$. 독미나리 & $\mathrm{CR}$ & 0 & & & & & \\
\hline 3 & Anagallidium dichotomum (L.) Grisb. 대성쓴풀 & $\mathrm{CR}$ & & & $\bigcirc$ & & & \\
\hline 4 & Cypripedium macranthon $\mathrm{Sw}$. 복주머니란* & $\mathrm{CR}$ & & & & & & $\bigcirc$ \\
\hline 5 & Daphne pseudomezereum var. koreana (Nakai) Hamaya 두메 닥나무* & EN & & & & $\bigcirc$ & & \\
\hline 6 & Trientalis europaea var. arctica (Fisch.) Ledeb. 기생꽃 & $\mathrm{EN}$ & $\bigcirc$ & & & & & \\
\hline 7 & Thuja koraiensis Nakai 눈측백* & VU & & & & $\bigcirc$ & & \\
\hline 8 & Taxus cuspidata Siebold \& Zucc. 주목 & VU & $\bigcirc$ & & & & & \\
\hline 9 & Leontice microrrhyncha S. Moore 한계령풀 & $\mathrm{VU}$ & $\bigcirc$ & & & & & \\
\hline 10 & Eleutherococcus senticosus (Rupr. \& Maxim.) Maxim. 가시오갈피 & VU & 0 & & & & & \\
\hline 11 & Monotropastrum humile (D. Don) Hara 나도수정초* & VU & & & & & & $\bigcirc$ \\
\hline 12 & Cynanchum inamoenum (Maxim.) Loes. 선백미꽃 & VU & & & $\bigcirc$ & & & \\
\hline 13 & Campanula glomerata var. dahurica Fisch. ex Ker Gawl. 자주꽃방망이 & VU & $\bigcirc$ & & & & $\bigcirc$ & \\
\hline 14 & Clintonia udensis Trautv. \& C.A. Mey. 나도옥잠화* & $\mathrm{VU}$ & & & & $\bigcirc$ & & \\
\hline 15 & Lilium callosum Siebold \& Zucc. 땅나리 & $\mathrm{VU}$ & $\bigcirc$ & $\bigcirc$ & & & & \\
\hline 16 & Lilium cernuum Kom. 솔나리 & VU & $\bigcirc$ & & $\bigcirc$ & & & \\
\hline 17 & Iris odaesanensis Y.N. Lee 노랑무늬붓꽃 & VU & $\bigcirc$ & & & & & \\
\hline 18 & Asplenium scolopendrium L. 골고사리* & LC & & & & & & O \\
\hline 19 & Anemone koraiensis Nakai 홀아비바람꽃 & $\mathrm{LC}$ & $\bigcirc$ & & & $\bigcirc$ & & $\bigcirc$ \\
\hline 20 & Clematis koreana Kom. 세잎종덩굴 & LC & $\bigcirc$ & & & $\bigcirc$ & $\bigcirc$ & $\bigcirc$ \\
\hline 21 & Eranthis stellata Maxim. 너도바람꽃 & LC & $\bigcirc$ & & & & & O \\
\hline 22 & Aristolochia manshuriensis Kom. 등칡 & LC & $\bigcirc$ & & & $\bigcirc$ & & \\
\hline 23 & Rodgersia podophylla A. Gray 도깨비부채* & LC & & & & $\bigcirc$ & & \\
\hline 24 & Viola albida Palib. 태백제비꽃 & $\mathrm{LC}$ & $\bigcirc$ & & & $\bigcirc$ & $\bigcirc$ & $\bigcirc$ \\
\hline 25 & Viola diamantiaca Nakai 금강제비꽃 & LC & $\bigcirc$ & $\bigcirc$ & & $\bigcirc$ & & \\
\hline 26 & Rhododendron brachycarpum D. Don ex G. Don 만병초* & $\mathrm{LC}$ & & & & $\bigcirc$ & & \\
\hline 27 & Syringa patula var. kamibayshii (Nakai) K. Kim 정 향나무* & $\mathrm{LC}$ & & & & $\bigcirc$ & & \\
\hline 28 & Syringa wolfii C.K. Schneid. 꽃개회나무 & $\mathrm{LC}$ & $\bigcirc$ & & & $\bigcirc$ & & \\
\hline 29 & Gentiana triflora var. japonica (Kusn.) H. Hara 과남풀* & LC & & & & $\bigcirc$ & & $\bigcirc$ \\
\hline 30 & Salvia chanryoenica Nakai 참배암차즈기 & LC & $\bigcirc$ & & & & & \\
\hline 31 & Parasenecio auriculatus (DC.) H. Koyama 귀박쥐나물* & LC & & & & O & & \\
\hline
\end{tabular}


Table 6. Continued.

\begin{tabular}{|c|c|c|c|c|c|c|c|c|}
\hline \multirow{2}{*}{ No. } & \multirow{2}{*}{ Scientific name } & \multirow{2}{*}{ Grade } & \multicolumn{6}{|c|}{ Locality } \\
\hline & & & 1 & 2 & 3 & 4 & 5 & 6 \\
\hline 32 & Parasenecio firmus (Kom.) Y.L. Chen 병풍쌈 & $\mathrm{LC}$ & $\bigcirc$ & & & & & \\
\hline 33 & Tephroseris flammea (Turcz.) Holub 산솜방망이 & $\mathrm{LC}$ & & $\bigcirc$ & & $\bigcirc$ & $\bigcirc$ & $\bigcirc$ \\
\hline 34 & Lilium distichum Nakai ex Kamibay 말나리* & $\mathrm{LC}$ & & & & $\bigcirc$ & & \\
\hline 35 & Lloydia triflora (Ledeb.) Baker 나도개감채 & $\mathrm{LC}$ & $\bigcirc$ & & $\bigcirc$ & & & O \\
\hline 36 & Streptopus ovalis (Ohwi) F.T. Wang \& Y.C. Tang 금강애기나리 & $\mathrm{LC}$ & $\bigcirc$ & & & $\bigcirc$ & & O \\
\hline 37 & Trillium kamtschaticum Pall. ex Pursh 연영초 & $\mathrm{LC}$ & $\bigcirc$ & & & $\bigcirc$ & & \\
\hline 38 & Iris ensata var. spontanea (Makino) Nakai 꽃창포 & $\mathrm{LC}$ & $\bigcirc$ & & & & & \\
\hline
\end{tabular}

1, Mt. Hambaek-san; 2, Mt. Geumdae-bong; 3, Mt. Daedeok-san; 4, Mt. Hambaek-san(Lee and Oh, 1999); 5, Mt. Hambaek-san(Oh et al., 2009); 6, Mt. Geumdae-bong(Oh et al., 2009); CR, Critically endangered species; EN, Endangered species; VU, Vulnerable species; LC, Least concerned species; *, Previous investigations.

Table 7. List of the rare and endangered plants (category II) in Mt. Hambaek-san and its neighboring mountains.

\begin{tabular}{cllc}
\hline \hline No. & Family & Scientific name / Korean name & 1 \\
\hline 1 & Ranunculaceae & Aconitum koreanum R. Raymund 백부자 & \\
2 & Berberidaceae & Leontice microrrhyncha S. Moore 한계령풀 & $\bigcirc$ \\
3 & Araliaceae & Eleutherococcus senticosus (Rupr. \& Maxim.) Maxim. 가시오갈피 & $\bigcirc$ \\
4 & Umbelliferae & Cicuta virosa L. 독미나리 & $\bigcirc$ \\
5 & Primulaceae & Trientalis europaea var. arctica (Fisch.) Ledeb. 기생꽃 & $\bigcirc$ \\
6 & Liliaceae & Lilium cernuum Kom. 솔나리 & $\bigcirc$ \\
7 & Iridaceae & Iris odaesanensis Y.N. Lee 노랑무늬붓꽃 & $\bigcirc$ \\
\hline
\end{tabular}

1, Mt. Hambaek-san; 2, Mt. Geumdae-bong; 3, Mt. Daedeok-san.

다. 대성쓴풀은 대덕산의 검룡소가 자생지로 알려져 있지 만(Kim and Park, 2009), 분주령 삼거리에서 보다 많은 개 체가 생육함을 확인하였고, 이는 검룡소 입구의 확장공사 로 인해 이전에 보고된 많은 개체가 소멸된 것으로 판단 된다. 한편, Oh et al. (2009)이 보고한 바 있는 복주머니란 은 금대봉 일대와 대덕산 검룡소-분주령 구간에서 생육이 확인되었으나, 이 구간은 자연생태보존지역으로 증거표 본 수집이 불가능하였기 때문에 전체 식물상 목록에서는 제외되었다. 그밖에 위기종 $(\mathrm{EN})$ 은 기생꽃 1 분류군, 취약 종(VU)은 주목(Taxus cuspidata Siebold \& Zucc.), 한계령풀 등의 8 분류군, 약관심종 $(\mathrm{LC})$ 은 홀아비바람꽃, 세잎종덩 굴(Clematis koreana Kom.) 등 14 분류군의 생육이 각각 확 인되었다. 한편, 증거표본이 제시되어 있는 선행연구 자 료를 포함하면 본 조사지역에 생육하는 희귀식물은 38 분 류군에 달한다(Table 6).

3) 멸종위기 야생식물 II등급

환경부 지정 멸종위기 야생식물(Ministry of Environment and National Institute of Environmental Research, 2006)에는 II 등급 종으로 백부자, 한계령풀, 가시오갈피[Eleutherococcus senticosus (Rupr. \& Maxim.) Maxim.], 독미나리, 기생꽃 등 7
분류군이 조사되었다(Table 7). 한계령풀은 다년초로서 뿌 리 끝에 덩이줄기가 달려있어 '메감자'라고도 불리며(Lee, 2003), 중부이북 고산지대에서 자란다고 알려져 있지만, 만항재 인근 활엽수림 하에 소수개체의 생육이 확인되었 으며, 가시오갈피는 약재로서 효능이 많이 알려져 야생개 체수가 줄어들고 있는 종으로 함백산 남사면 혈동 지역에 서 소수개체가 발견되었다. 본 조사에서 확인된 멸종위기 식물의 자생지는 일부종을 제외하고, 전반적으로 등산로 와 같이 직접적인 인간 활동의 영향권에서 벗어나 있지만, 일부 지역은 고랭지 밭과 광산의 채굴작업을 위한 산림훼 손과 많은 등산객에 의한 인위적인 환경파괴가 우려된다.

\section{4) 식물구계학적특정식물종}

조사지역의 식물구계학적특정종 $(\mathrm{Kim}, 2000$; Ministry of Environment and National Institute of Environmental Research, 2006)은 I, II등급을 제외하고 총 62분류군으로 나타났다. 이를 등급별로 살펴보면 $\mathrm{V}$ 등급은 좀우드풀 (Woodsia intermedia Tagawa), 백부자, 한계령풀, 가시오갈피, 기생꽃, 솔나리(Lilium cernuum Kom.) 등의 8분류군, IV등급 은 홀아비바람꽃, 생열귀나무(Rosa davurica Pall.), 각시괴 불나무(Lonicera chrysantha Turcz.), 큰제비란(Platanthera 
sachalinensis F. Schmidt) 등 21분류군, III등급은 검종덩굴, 금강제비꽃, 가지더부살이(Phacellanthus tubiflorus Siebold $\&$ Zucc.), 연복초(Adoxa moschatellina L.) 등 33분류군이 각 각 확인되었으며(Table 8), 이는 함백산 일원의 관속식물 622 분류군의 $9.96 \%$ 를 차지하였다. 한편, 함백산에서 중함 백으로 이어지는 능선에 위치한 주목(Taxus cuspidata Siebold \& Zucc.) 자생지는 산림청에서 보호지역으로 지정 하였지만, 보호지역을 구분하는 울타리 이외에 별도의 보 호수단이 없어 탐방객들의 휴식처로 흔히 이용되고 있으 며, 상당수가 고사되고 있다. 또한, 이곳은 주목과 구상나 무(Abies koreana Wilson)가 자주 출현하고, 아교목-관목층 으로는 당단풍나무, 개비자나무(Cephalotaxus koreana Nakai), 노린재나무[Symplocos chinensis f. pilosa (Nakai) Ohwi], 고로쇠나무, 복장나무, 시닥나무(Acer komarovii Pojark.), 자작나무, 피나무, 매발톱나무(Berberis amurensis
Rupr.), 철쭉(Rhododendron schlippenbachii Maxim.), 산철쭉 [Rhododendron yedoense f. poukhanense (H. Lév.) Sugim.] 등 이 생육하고 있으며, 태백기린초(Sedum latiovalifolium Y.N. Lee), 금강제비꽃, 기생꽃, 노랑무늬붓꽃 등의 희귀 또는 특산 식물이 다수 분포하고 있어 보호지역을 확대하 고, 이에 대한 자생지 보호대책이 필요하다고 판단된다.

\section{5) 귀화식물}

조사지역에서 확인된 귀화식물은 10 과 30 분류군이며 (Table 9), 귀화율은 7.7\%로 나타났다. 인근 산지의 귀화율 인 청옥산과 두타산(Cho et al., 1999) $2.9 \%$, 백운산(Song, 2000) $5.6 \%$, 매봉산(Hong, 2000) $3.5 \%$, 덕항산(Kim et al., 2005 ) $3.2 \%$, 괘병산과 갈미봉 (Kim et al., 2012) $3.6 \%$ 보다 높 은 수치이다. 또한, 도시화지수 역시 함백산 일대는 $6.7 \%$ 로 나타나, 청옥산과 두타산 $6.2 \%$, 매봉산 $6.7 \%$, 덕항산

Table 8. Number of the floristically specific taxa designated as Grade I-V (Ministry of Environment and National Institute of Environmental Research, 2006) found in this investigation.

\begin{tabular}{ccccccc}
\hline \hline Mountain & V & IV & III & II & I & Total \\
\hline Mt. Hambaek-san & 5 & 20 & 30 & 40 & 40 & 135 \\
Mt. Geumdae-bong & 1 & 3 & 5 & 9 & 8 & 26 \\
Mt. Daedeok-san & 2 & 6 & 9 & 20 & 17 & 54 \\
\hline Total & 8 & 21 & 33 & 44 & 43 & 149 \\
\hline
\end{tabular}

Table 9. List of the naturalized plants in Mt. Hambaek-san and its neighboring mountains.

\begin{tabular}{|c|c|c|c|c|c|}
\hline No. & Family & Scientific name & 1 & 2 & 3 \\
\hline 1 & Cannabaceae & Cannabis sativa L. 삼 & $\bigcirc$ & & \\
\hline 2 & Polygonaceae & Fallopia dumetorum (L.) Holub 닭의덩굴 & $\bigcirc$ & & \\
\hline 3 & Polygonaceae & Rumex crispus L. 소리쟁이 & $\bigcirc$ & & \\
\hline 4 & Caryophyllaceae & Silene armeria L. 끈끈이대나물 & $\bigcirc$ & & \\
\hline 5 & Chenopodiaceae & Chenopodium glaucum L. 취명아주 & & & 0 \\
\hline 6 & Cruciferae & Thlaspi arvense L. 말냉이 & $\bigcirc$ & & \\
\hline 7 & Leguminosae & Astragalus sinicus L. 자운영 & 0 & & O \\
\hline 8 & Leguminosae & Medicago lupulina L. 잔개자리 & $\bigcirc$ & & 0 \\
\hline 9 & Leguminosae & Medicago polymorpha L. 개자리 & $\bigcirc$ & & \\
\hline 10 & Leguminosae & Melilotus alba Medicus 흰전동싸리 & $\bigcirc$ & & \\
\hline 11 & Leguminosae & Melilotus suaveolens Ledeb. 전동싸리 & $\bigcirc$ & & O \\
\hline 12 & Leguminosae & Trifolium pratense L. 붉은토끼풀 & $\bigcirc$ & & $\bigcirc$ \\
\hline 13 & Leguminosae & Trifolium repens L. 토끼풀 & & & 0 \\
\hline 14 & Onagraceae & Oenothera biennis L. 달맞이꽃 & & & O \\
\hline 15 & Boraginaceae & Symphytum officinale L. 컴프리 & $\bigcirc$ & & \\
\hline 16 & Compositae & Achillea millefolium L. 서양톱풀 & $\bigcirc$ & & $\bigcirc$ \\
\hline 17 & Compositae & Bidens frondosa L. 미국가막사리 & $\bigcirc$ & & \\
\hline 18 & Compositae & Carduus crispus L. 지느러미엉겅퀴 & $\bigcirc$ & & $\bigcirc$ \\
\hline 19 & Compositae & Conyza canadensis (L.) Cronquist 망초 & & & 0 \\
\hline
\end{tabular}


Table 9. Continued.

\begin{tabular}{cllll}
\hline \hline No. & Family & \multicolumn{1}{c}{ Scientific name } & 1 & 2 \\
\hline 20 & Compositae & Erigeron annuus (L.) Pers. 개망초 & $\bigcirc$ & $\bigcirc$ \\
21 & Compositae & Matricaria matricariodes Porter 족제비쑥 & & \\
22 & Compositae & Senecio vulgaris L. 개쑥갓 & $\bigcirc$ & \\
23 & Compositae & Solidago serotina Aiton 미국미역취 & $\bigcirc$ & $\bigcirc$ \\
24 & Compositae & Taraxacum officinale Weber 서양민들레 & $\bigcirc$ & $\bigcirc$ \\
25 & Gramineae & Alopecurus pratensis L. 큰뚝새풀 & $\bigcirc$ \\
26 & Gramineae & Bromus unioloides Kunth 큰이삭풀 & $\bigcirc$ & $\bigcirc$ \\
27 & Gramineae & Dactylis glomerata L. 오리새 & & \\
28 & Gramineae & Lolium perenne L. 호밀풀 & $\bigcirc$ & $\bigcirc$ \\
30 & Gramineae & Panicum dichotomiflorum Michx. 미국개기장 & & $\bigcirc$ \\
\hline
\end{tabular}

1, Mt. Hambaek-san; 2, Mt. Geumdae-bong; 3, Mt. Daedeok-san.

Table 10. Use types of vascular plants in Mt. Hambaek-san and its neighboring mountains.

\begin{tabular}{ccccccc}
\hline \hline & $\mathrm{E}$ & $\mathrm{M}$ & $\mathrm{P}$ & $\mathrm{I}$ & $\mathrm{O}$ & $\mathrm{T}$ \\
\hline Number of taxa & 222 & 180 & 211 & 2 & 68 & 14 \\
Ratio (\%) & 35.6 & 28.9 & 33.9 & 0.3 & 10.9 & 2.2 \\
\hline
\end{tabular}

E, Edible plant; M, Medicinal plant; P, Pasture plant; I, Industrial raw material plant O, Ornamental plant; T, Timber source plant.

$5.9 \%$, 괘병산과 갈미봉 $5.3 \%$ 보다 비슷하거나 비교적 높은 수치를 보였다. 이는 다른 산지에 비해 교통이 편리하고, 등산객의 수가 상대적으로 많아 인위적인 교란이 심했기 때문으로 판단되며, 귀화식물의 분포역과 분포특성 등을 파악하여 귀화식물에 대한 지속적인 관리와 대책이 필요 하다.

\section{6) 용도별 구분}

본 조사에서 생육이 확인된 622분류군을 대상으로 용도 별 자원식물 현황(Lee, 1976)을 분석한 결과, 464분류군이 자원식물로서 이용가치가 있는 것으로 나타났다. 이들을 용도에 따라 구분해보면, 식용식물(E) 222분류군(35.6\%), 약용식물(M) 180 분류군(28.9\%), 목초용식물(P) 211분류군 (33.9\%), 공업용식물(I) 2분류군 $(0.3 \%)$, 관상용식물 $(\mathrm{O}) 68$ 분류군(10.9\%), 목재용식물(T) 14 분류군(2.2\%)로 나타났 다(Table 10; Appendix 2).

\section{식물지리학적 특성}

함백산 일원에서 조사된 622분류군에 대한 분포지 조사 결과 북부고유분자는 붉은터리풀, 긴생열귀(Rosa davurica var. ellipsoidea Nakai), 무산상자[Sphallerocarpus gracilis (Besser) Koso-Pol.], 개씀배(Prenanthes tatarinowii Maxim.), 갈색사초(Carex caryophyllea var. microtricha Kük.), 함북사 초(Carex echinata Murr)의 총 6분류군, 중부고유분자는 홀 아비바람꽃, 요강나물, 태백이질풀, 대성쓴풀 등 13분류
군, 남부고유분자는 산비늘고사리[Dryopteris polylepis (Franch. \& Sav.) C. Chr.], 털고광나무(Philadelphus schrenkii var. jackii Koehne) 등 4분류군으로 중부고유분자가 가장 많았다(Appendix 2). 한편, 중북부공통분자는 130 분류군, 남중부공통분자는 82 분류군으로 중북부공통분자의 분포 가 높은 수치를 보임으로써, 본 조사결과는 Nakai (1935)와 Lee and $\operatorname{Yim}(1978,2002)$ 의 한반도 식생의 구계구분에 의 한 중부아구와 일치함을 보였고, 중북부공통분자가 남중 부공통분자보다 더 높게 나타난 것으로 볼 때, 본 조사구 역은 전형적인 중부고유식생을 보이나 북부분자의 식물 이 더 많이 유입된 지역이라고 판단된다.

\section{금대봉-대덕산 생태계 보존지역}

본 조사지역의 금대봉-대덕산 일대는 생태계 보존 가치 가 높아 환경부로부터 1993년에 생태 - 경관 보존지역으로 지정되었다. 본 조사에서는 복주머니란, 대성쓴풀, 노랑 투구꽃(Aconitum sibiricum Poir.), 산솜방망이, 노랑무늬붓 꽃, 홀아비바람꽃 등의 희귀식물의 생육을 확인하였으나, 증거표본으로의 수집은 불가능하였다. 보존지역 중 검룡 소-분주령 구간의 초입은 등산로 확장 공사로 인하여 대 성쓴풀 자생지가 거의 소멸된 상태임을 제외하고는 전체 적 비교적 잘 보존 - 관리되고 있다고 판단된다. 이는 본 지 역이 많은 탐방객이 찾는 장소임에도 불구하고 무분별한 입산행위를 제한하기 위해 사전 예약제를 통하여 관리되 고 있기 때문으로 생각된다. 


\section{사 사}

본 연구는 환경부 국립생물자원관(NIBR No. 2014-02001)과 산림청 연구과제, ‘우리 식물의 주권확보(CBD 등) 를 위한 기반연구'No. S111114L040100)의 지원에 의하여 수행되었습니다.

\section{Literature Cited}

Cho, C. G., W. G. Paik and W. T. Lee. 1999. Flora and Vegetation of the Southern Slope Area at Mt. Chongok and Mt. Tuta(Kangwon-do). Korean Journal of Plant Resource 12(3): 240-252. (in Korean)

Hong, M. G. 2000. Phytosociological Study on the Forest Vegetation of Mt. Maebong and Mt. Chiljelbong. MS thesis, Konkuk University, Seoul. Pp. 1-72. (in Korean)

Jeongseon county. 2012. Statistical Yearbook. Retrieved Oct. 7, 2014, from http://www.Jeongseon.go.kr.

Kang, S. O. 1971. New Korea Geography. Saegeulsa, Seoul. (in Korean)

Kim, C. H. 2000. Assessment of natural environment: I. Selection of plants taxa. Korean Journal of Environmental Biology 18: 163-198. (in Korean)

Kim, C. N. 2001. Flora and Forest Vegetation of Mt. Hambaek. Journal of The Korea Association for Research in Science Education 6(1): 75-87. (in Korean)

Kim, G. T., G. C. Choo and G. J. Baek. 2004. Structure of Forest Community at Daedeoksan-Geumdaebong Nature Ecosystem Preservation Area in Baekdudaegan. Korean Society of Environment and Ecology 17(1): 9-17. (in Korean)

Kim, J. S., B. C. Lee, J. M. Chung and J. H. Park. 2005. Flora and Phytogeography on Mt. Deokhang (Gangwon-do). Korean Journal of Plant Taxonomy 35(4): 337-364. (in Korean)

Kim, T. H and C. S. Park. 2009. The wild flower in Taebaek. Singubook, Seoul. (in Korean)

Kim, Y. D and Y. I. Park. 2012. Flora of Mt. Hambaek. 3rd. National Natural environment survey. Ministry of Environment, Incheon, Korea. Pp. 1-6. (in Korean)

Kim, Y. S., N. Y. Kim, Y. S. Kim, H. B. Lee, S. C. Kim, J. H. Kim and W. G. Park. 2012. Flora and Vegetation of Mt. Gwaebyung and Galmi-bong. Forest science and technology 101(2): 226-235. (in Korean)

Korea Forest service and Korea National Arboretum. 2008. Rare Plants Data Book in Korea. Korea National Arboretum, Pocheon. (in Korean)

Korea National Arboretum and The Plant Taxonomic Society of Korea. 2007. A Synonymic List of Vascular Plants in Korea. Korea National Arboretum, Pocheon. (in Korean)
Korean Meterological Administration. 2008. Annual Report of Automatic Weather Station Data. Korean Meterological Administration, Seoul. (in Korean)

Korean Meterological Administration. 2009. Annual Report of Automatic Weather Station Data. Korean Meterological Administration, Seoul. (in Korean)

Korean Meterological Administration. 2010. Annual Report of Automatic Weather Station Data. Korean Meterological Administration, Seoul. (in Korean)

Korean Meterological Administration. 2011. Annual Report of Automatic Weather Station Data. Korean Meterological Administration, Seoul. (in Korean)

Korean Meterological Administration. 2012. Annual Report of Automatic Weather Station Data. Korean Meterological Administration, Seoul. (in Korean)

Lee, T. B. 1976. Vascular Plants and their uses in Korea. Bulletin of the Arboretum Seoul National University 1: 1-131.

Lee, T. B. 1980. Illustrated Flora of Korea. Hyangmunsa, Seoul. (in Korean)

Lee, T. B. 2003. Coloured Flora of Korea. Hyangmunsa, Seoul. (in Korean)

Lee, W. T and Y. J. Oh. 1999. Flora of Mt. Hambaek area. 2nd. National Natural environment survey. Ministry of Environment, Incheon, Korea. Pp. 145-179. (in Korean)

Lee, W. T. 1987 Flora of Mt. Taebaek. The report of the KACN 25: 43-57. (in Korean)

Lee, W. T. and Y. J. Yim. 1978. Studies on the distribution of Vascular plants in the Korean Peninsula. Korean Journal of Plant Taxonomy 8 (Appendix): 1-33. (in Korean)

Lee, W. T. and Y. J. Yim. 2002. Plant Geography. Kangwon National University Press. Chuncheon. (in Korean)

Lee, Y. M., S. H. Park, S. Y. Jung, S. H. Oh and J. C. Yang. 2011. Study on the current status of naturalized plants in South Korea. Korean Journal of Plant Taxonomy 41(1): 87-101. (in Korean)

Lee, Y. N. 1996. Flora of Korea, Gyohaksa, Seoul. (in Korean)

Lee, Y. N. 2006. New Flora of Korea. Gyohaksa, Seoul. (in Korean)

Melchior, H. 1964. An Engler's Syllabus der pflanzenfamilien. Band II. Gebruder Borntraeger, Berlin.

Ministry of Environment and National Institute of Environmental Research. 2006. Third Round of the National Natural Environment Research Guidelines. Ministry of Environment and National Institute of Environmental Research, Gwacheon and Incheon. (in Korean)

Nakai, T. 1935. Some Noticeable Japanese Plant (I). Journal of Japanese Botany 11: 147-156.

Oh, B. U., D. G. Jo, K. S. Kim and C. G. Jang. 2005. Endemic Vas- 
cular Plants in the Korean Peninsula (1st Step Result). Korea National Arboretum, Pocheon. (in Korean)

Oh, B. U., D. G. Jo, S. C. Ko, H. T. Im, G. Y. Chung, C. G. Jang and S. H. Kang. 2009. Distribution Maps of Vascular Plants of Korean Peninsula. VI. Middle Province(Gangwon-do). Oh, B. U. (ed.), Korean Forest Service and Korean National Arboretum, Pocheon. (in Korean)

Park, C. W. 2007. The genera of vascular plants of Korea. Park, C.W. (ed.), Academy Publishing Co., Seoul.

Park, I. H., Y. H. Choi, S. M. Lee, Y. C. Choi and S. B. Ryu. 2002. Forest Structure in Relation to Slope Aspect and Altitude in Valley Forests at Hambaeksan Area. Korean Journal of Environment and Ecology 15(4): 361-368. (in Korean)
Park, S. H. 2009. New Illustrations and Photographs of Naturalized Plants of Korea. Ilchokak, Seoul. (in Korean)

Seo, D. J., W. K. Park, W. T. Lee and K. O. Yoo. 2006. Flora and Vegetation of Mt. Daeduk. Korean Journal of Plant Resources 19(2): 277-295. (in Korean)

Song, E. G. 2000. Flora and Forest Vegetation of Mt. Baekun (Gangwon-do). MS thesis, Gangwon National University, Samcheok. Pp. 1-42. (in Korean)

Taebaek city. 2012. Statistical Yearbook. Retrieved Oct. 7, 2014, from http://www.Taebaek.go.kr. 
Appendix 1. List of the vascular plants of Mt. Hambaek-san and its neighboring mountains.

\begin{tabular}{|c|}
\hline Scientific name \\
\hline $\begin{array}{l}\text { Lycopodiaceae 석송과 } \\
\star 1 \text {. Lycopodium clavatum L. 석송 } \\
\text { Jeongseon-gun(Gohan-ri, Mt. Hambaek)-130502-009 [HNHM] }\end{array}$ \\
\hline $\begin{array}{l}\text { Equisetaceae 속새과 } \\
\text { 2. Equisetum arvense L. 쇠뜨기 } \\
\text { Jeongseon-gun(Gohan-ri, Mt. Hambaek)-130411-012 [HNHM] }\end{array}$ \\
\hline $\begin{array}{l}\star 3 . \text { Equisetum hyemale L. 속새 } \\
\text { Taebaek-si(Hyeol-dong, Mt. Hambaek)-140605-135 [HNHM] }\end{array}$ \\
\hline $\begin{array}{l}\text { Ophioglossaceae 고사리삼과 } \\
\text { ^4. Sceptridium ternatum (Thunb.) Lyon 고사리삼 } \\
\text { Jeongseon-gun(Gohan-ri, Mt. Hambaek)-131003-070 [KB] }\end{array}$ \\
\hline $\begin{array}{l}\text { Dennstaedtiaceae 잔고사리과 } \\
\text { ^5. Dennstaedtia wilfordii (Moore) Christ 황고사리 } \\
\text { Taebaek-si(Hyeol-dong, Mt. Hambaek)-140704-101 [HNHM] }\end{array}$ \\
\hline $\begin{array}{l}\text { Parkeriaceae 공작고사리과 } \\
\text { ^6. Coniogramme intermedia Hieron 고비고사리 } \\
\text { Taebaek-si(Hyeol-dong, Mt. Hambaek)-140704-098 [HNHM] }\end{array}$ \\
\hline $\begin{array}{l}\text { Aspleniaceae 꼬리고사리과 } \\
\text { ^7. Asplenium ruprechtii Kurata 거미고사리 } \\
\text { Taebaek-si(Hyeol-dong, Mt. Hambaek)-140605-226 [HNHM] }\end{array}$ \\
\hline $\begin{array}{l}\text { Dryopteridaceae 면마과 } \\
\text { 8. Arachniodes borealis Serizawa 왁살고사리 } \\
\text { Jeongseon-gun(Gohan-ri, Mt. Hambaek)-130828-272 [KB] }\end{array}$ \\
\hline $\begin{array}{l}\star \text { `. Dryopteris chinensis (Bak.) Koidz. 가는잎족제비고사리 } \\
\text { Jeongseon-gun(Gohan-ri, Mt. Hambaek)-120703-020 [KB] }\end{array}$ \\
\hline $\begin{array}{l}\text { 10. Dryopteris crassirhizoma Nakai 관중 } \\
\text { Jeongseon-gun(Gohan-ri, Mt. Hambaek)-120524-293 [KB] }\end{array}$ \\
\hline $\begin{array}{l}\star 11 . \text { Dryopteris lacera (Thunb.) Kuntze 비늘고사리 } \\
\text { Jeongseon-gun(Gohan-ri, Mt. Hambaek)-130412-016 [KB] }\end{array}$ \\
\hline $\begin{array}{l}\text { 12. Dryopteris polylepis (Franch. \& Sav.) C. Chr. 산비늘고사리 } \\
\text { Samcheok-si(Hanso-ri, Mt. Daedeok)-130710-009 [KB] }\end{array}$ \\
\hline $\begin{array}{l}\text { 13. Polystichum tripteron (Kunze) C. Pres1 십자고사리 } \\
\text { Taebaek-si(Changjuk-dong, Mt. Geumdae)-120704-059 [KB] }\end{array}$ \\
\hline $\begin{array}{l}\text { Woodsiaceae 우드풀과 } \\
\text { 14. Athyrium brevifrons Kodama ex Nakai 참새 발고사리 } \\
\text { Taebaek-si(Hyeol-dong, Mt. Hambaek)-140704-283 [HNHM] }\end{array}$ \\
\hline $\begin{array}{l}\star 15 \text {. Athyrium iseanum Rosenst. 가는잎개고사리 } \\
\text { Jeongseon-gun(Gohan-ri, Mt. Hambaek)-131003-031 [KB] }\end{array}$ \\
\hline $\begin{array}{l}\text { 16. Athyrium niponicum (Mett.) Hance 개고사리 } \\
\text { Taebaek-si(Hyeol-dong, Mt. Hambaek)-140705-239 [HNHM] }\end{array}$ \\
\hline $\begin{array}{l}\star \text { 17. Athyrium vidalii (Franch. \& Sav.) Nakai 산개고사리 } \\
\text { Jeongseon-gun(Gohan-ri, Mt. Hambaek)-130828-034 [KB] }\end{array}$ \\
\hline $\begin{array}{l}\text { 18. Onoclea orientalis (Hk.) Hk. 개면마 } \\
\text { Jeongseon-gun(Gohan-ri, Mt. Hambaek)-131003-095 [KB] }\end{array}$ \\
\hline $\begin{array}{l}\star 19 . \text { Woodsia intermedia Tagawa 좀우드풀 } \\
\text { Taebaek-si(Hyeol-dong, Mt. Hambaek)-140705-333 [HNHM] }\end{array}$ \\
\hline $\begin{array}{l}\text { 20. Woodsia polystichoides D.C. Eaton 우드풀 } \\
\text { Samcheok-si(Panmun-ri, Mt. Daedeok)-120718-222 [KB] }\end{array}$ \\
\hline $\begin{array}{l}\text { Pinaceae 소나무과 } \\
\text { 21. Larix kaempferi (Lamb.) Carriére 일본잎갈나무 } \\
\text { Taebaek-si(Hyeol-dong, Mt. Hambaek)-140704-008 [HNHM] }\end{array}$ \\
\hline $\begin{array}{l}\text { 22. Pinus koraiensis Siebold \& Zucc. 잣나무 } \\
\text { Taebaek-si(Hyeol-dong, Mt. Hambaek)-140704-383 [HNHM] }\end{array}$ \\
\hline $\begin{array}{l}\text { Cephalotaxaceae 개비자나무과 } \\
\text { ^23. Cephalotaxus koreana Nakai 개비자나무 } \\
\text { Taebaek-si(Hyeol-dong, Mt. Hambaek)-140704-178 [HNHM] }\end{array}$ \\
\hline Taxaceae 주목과 \\
\hline
\end{tabular}

24. Taxus cuspidata Siebold \& Zucc. 주목

Jeongseon-gun(Gohan-ri, Mt. Hambaek)-130828-122 [KB]

$\star 25$. Torreya nucifera (L.) Siebold \& Zucc. 비자나무

Jeongseon-gun(Gohan-ri, Mt. Hambaek)-140425-037 [HNHM]

Juglandaceae 가래나무과

26. Juglans mandshurica Maxim. 가래나무

Taebaek-si(Changjuk-dong, Mt. Daedeok)-120525-244 [KB]

Salicaceae 버드나무과

27. Populus davidiana Dode 사시나무

Taebaek-si(Hwajeon-dong, Mt. Eundae)-140807-073 [HNHM]

28. Salix caprea L. 호랑버들

Jeongseon-gun(Gohan-ri, Mt. Hambaek)-120524-311 [KB]

29.Salix gracilistyla Miq. 갯버들

Jeongseon-gun(Gohan-ri, Mt. Hambaek)-120418-011 [KB]

30. Salix koriyanagi Kimura 키버들

Jeongseon-gun(Gohan-ri, Mt. Hambaek)-130502-025 [HNHM]

$\star 31$. Salix rorida Laksch. 분버들

Jeongseon-gun(Gohan-ri, Mt. Hambaek)-130501-134 [HNHM]

Betulaceae 자작나무과

32. Alnus sibirica Fisch. ex Turcz. 물오리나무

Taebaek-si(Hyeol-dong, Mt. Hambaek)-140705-101 [HNHM]

33. Betula davurica Pall. 물박달나무

Taebaek-si(Hwajeon-dong, Mt. Eundae)-140807-140 [HNHM]

$\star$ 34. Betula platyphylla var. japonica (Miq.) Hara 자작나무

Taebaek-si(Hyeol-dong, Mt. Hambaek)-140705-280 [HNHM]

35. Betula schmidtii Regel 박달나무

Taebaek-si(Hyeol-dong, Mt. Hambaek)-140705-019 [HNHM]

36. Carpinus cordata Blume 까치박달

Jeongseon-gun(Gohan-ri, Mt. Hambaek)-130711-035 [KB]

^37. Corylus heterophylla Fisch. ex Trautv. 개암나무

Taebaek-si(Hwajeon-dong, Mt. Eundae)-140807-239 [HNHM]

38. Corylus sieboldiana var. mandshurica C.K. Schneid. 물개암나무

Taebaek-si(Hyeol-dong, Mt. Hambaek)-140704-035 [HNHM]

Fagaceae 참나무과

39. Quercus mongolica Fisch. ex Ledeb. 신갈나무

Jeongseon-gun(Baekjeon-ri, Mt. Daedeok)-120814-188 [KB]

Ulmaceae 느릅나무과

40. Ulmus davidiana var. japonica (Rehder) Nakai 느릅나무

Taebaek-si(Hyeol-dong, Mt. Hambaek)-140705-249 [HNHM]

$\star 41$. Ulmus laciniata (Trautv.) Mayr 난티나무

Taebaek-si(Hyeol-dong, Mt. Hambaek)-140704-114 [HNHM]

Moraceae 뽕나무과

$\star$ 42. Morus bombycis Koidz. 산뽕나무

Jeongseon-gun(Baekjeon-ri, Mt. Daedeok)-140523-098 [HNHM]

Cannabaceae 삼과

$\star$ 43. Cannabis sativa L. 삼

Jeongseon-gun(Gohan-ri, Mt. Hambaek)-120705-004 [KB]

44. Humulus japonicus Siebold \& Zucc. 환삼덩굴

Taebaek-si(Hwajeon-dong, Mt. Eundae)-140807-190 [HNHM]

Urticaceae 쐐기풀과

45. Boehmeria longispica Steud. 왜모시풀

Taebaek-si(Hyeol-dong, Mt. Hambaek)-140704-199 [HNHM]

$\star$ 46. Boehmeria platanifolia Franch. \& Sav. 개모시풀

Jeongseon-gun(Gohan-ri, Mt. Hambaek)-120705-107 [KB]

$\star$ 47. Boehmeria spicata (Thunb.) Thunb. 좀깨잎나무

Taebaek-si(Hyeol-dong, Mt. Hambaek)-140705-098 [HNHM]

48. Laportea bulbifera (Siebold \& Zucc.) Wedd. 혹쐐기풀

Taebaek-si(Changjuk-dong, Mt. Daedeok)-120920-302 [KB]

$\star$ 49. Pilea hamaoi Makino 큰물통이

Jeongseon-gun(Gohan-ri, Mt. Hambaek)-130828-015 [KB]

50. Pilea mongolica Wedd. 모시물통이 
Jeongseon-gun(Gohan-ri, Mt. Hambaek)-131003-051 [KB] 51. Urtica angustifolia Fisch. ex Hornem. 가는잎쐐기풀 Taebaek-si(Hyeol-dong, Mt. Hambaek)-140704-139 [HNHM]

$\star 52$. Urtica laetevirens Maxim. 애기쐐기풀

Taebaek-si(Hyeol-dong, Mt. Hambaek)-140704-010 [HNHM]

$\star 53$. Urtica thunbergiana Siebold \& Zucc. 쐐기풀

Taebaek-si(Hyeol-dong, Mt. Hambaek)-140704-115 [HNHM]

Loranthaceae 꼬리겨우살이과

$\star 54$. Viscum album var. coloratum (Kom.) Ohwi 겨우살이

Samcheok-si(Hanso-ri, Mt. Daedeok)-130710-325 [KB]

Polygonaceae 마디풀과

$\star 55$. Bistorta incana (Nakai) Nakai ex Mori 흰범꼬리 Jeongseon-gun(Gohan-ri, Mt. Hambaek)-140705-244 [HNHM]

56. Bistorta manshuriensis (Petrov ex Kom.) Kom. 범꼬리 Jeongseon-gun(Gohan-ri, Mt. Hambaek)-130711-140 [KB]

57. Fagopyrum esculentum Moench 메밀

Jeongseon-gun(Baekjeon-ri, Mt. Daedeok)-120814-047 [KB]

58. Fallopia dumetorum (L.) Holub 닭의덩굴

Taebaek-si(Hyeol-dong, Mt. Hambaek)-140705-242 [HNHM]

$\star$ 59. Persicaria dissitiflora (Hemsl.) H. Gross ex Mori 가시여뀌

Taebaek-si(Hwajeon-dong, Mt. Eundae)-140807-131 [HNHM]

$\star 60$. Persicaria hydropiper (L.) Spach 여뀌

Jeongseon-gun(Gohan-ri, Mt. Hambaek)-120919-009 [KB]

61. Persicaria japonica (Meisn.) H. Gross ex Nakai 흰꽃여뀌 Jeongseon-gun(Baekjeon-ri, Mt. Daedeok)-120814-128 [KB]

62. Persicaria lapathifolia (L.) Gray 흰여뀌

Jeongseon-gun(Gohan-ri, Mt. Hambaek)-131003-071 [KB]

63. Persicaria longiseta (Bruijn) Kitag. 개여뀌

Taebaek-si(Hwajeon-dong, Mt. Eundae)-140807-100 [HNHM]

64. Persicaria nepalensis (Meisn.) H. Gross 산여뀌

Taebaek-si(Hwajeon-dong, Mt. Eundae)-140807-065 [HNHM]

$\star$ 65. Persicaria posumbu var. laxiflora (Meisn.) H. Hara 장대여뀌

Taebaek-si(Hwajeon-dong, Mt. Eundae)-140807-050 [HNHM]

66. Persicaria sagittata (L.) H. Gross ex Nakai 미꾸리낚시

Taebaek-si(Hwajeon-dong, Mt. Eundae)-140807-084 [HNHM]

67. Persicaria senticosa (Meisn.) H. Gross ex Nakai 며느리밑씻개

Taebaek-si(Hyeol-dong, Mt. Hambaek)-140704-144 [HNHM]

68. Persicaria thunbergii (Siebold \& Zucc.) H.. Gross ex Nakai 고마리 Jeongseon-gun(Gohan-ri, Mt. Hambaek)-130828-045 [KB]

69. Persicaria vulgaris Webb \& Moq. 봄여뀌

Jeongseon-gun(Baekjeon-ri, Mt. Daedeok)-120814-232 [KB]

70. Polygonum aviculare L. 마디풀

Samcheok-si(Panmun-ri, Mt. Daedeok)-120718-243 [KB]

$\star 71$. Rumex conglomeratus Murray 묵밭소리쟁이

Taebaek-si(Hwajeon-dong, Mt. Eundae)-140807-194 [HNHM]

72. Rumex crispus L. 소리쟁이

Taebaek-si(Hyeol-dong, Mt. Hambaek)-140704-248 [HNHM]

Caryophyllaceae 석죽과

$\star$ 73. Arenaria serpyllifolia L. 벼룩이자리

Taebaek-si(Hyeol-dong, Mt. Hambaek)-140705-270 [HNHM]

74. Cerastium holosteoides var. hallaisanense (Nakai) Mizush. 점나도나물

Jeongseon-gun(Gohan-ri, Mt. Hambaek)-140522-109 [HNHM]

$\star 75$. Cucubalus baccifer var. japonicus Miq. 덩굴별꽃

Taebaek-si(Hyeol-dong, Mt. Hambaek)-140704-110 [HNHM]

$\star 76$. Dianthus longicalyx Miq. 술패랭이꽃

Jeongseon-gun(Gohan-ri, Mt. Hambaek)-131003-082 [KB]

77. Lychnis cognata Maxim. 동자꽃

Jeongseon-gun(Gohan-ri, Mt. Hambaek)-130711-134 [KB]

$\star 78$. Pseudostellaria coreana (Nakai) Ohwi 참개별꽃

Taebaek-si(Hyeol-dong, Mt. Hambaek)-140704-024 [HNHM]

79. Pseudostellaria davidii (Franch.) Pax ex Pax \& Hoffm. 덩굴개별꽃
Jeongseon-gun(Gohan-ri, Mt. Hambaek)-140522-116 [HNHM]

80. Pseudostellaria heterophylla (Miq.) Pax ex Pax \& Hoffm. 개별꽃 Jeongseon-gun(Gohan-ri, Mt. Eundae)-140424-017 [HNHM]

81. Pseudostellaria palibiniana (Takeda) Ohwi 큰개별꽃

Jeongseon-gun(Gohan-ri, Mt. Hambaek)-140522-040 [HNHM]

$\star 82$. Silene armeria L. 끈근이대나물

Taebaek-si(Hyeol-dong, Mt. Hambaek)-140705-347 [HNHM]

83. Silene firma Siebold \& Zucc. 장구채

Taebaek-si(Hwajeon-dong, Mt. Eundae)-140807-085 [HNHM]

84. Stellaria aquatica (L.) Scop. 쇠별꽃

Samcheok-si(Hanso-ri, Mt. Daedeok)-130710-286 [KB]

$\star 85$. Stellaria media (L.) Vill. 별꽃

Jeongseon-gun(Gohan-ri, Mt. Hambaek)-131003-030 [KB]

Chenopodiaceae 명아주과

86. Chenopodium glaucum L. 취명아주

Jeongseon-gun(Baekjeon-ri, Mt. Daedeok)-121024-008 [KB]

Amaranthaceae 비름과

87. Amaranthus mangostanus L. 비름

Taebaek-si(Changjuk-dong, Mt. Daedeok)-120920-140 [KB]

Magnoliaceae 목련과

88. Magnolia sieboldii K. Koch 함박꽃나무

Jeongseon-gun(Baekjeon-ri, Mt. Daedeok)-140523-089 [HNHM]

Schisandraceae 오미자과

89. Schisandra chinensis (Turcz.) Baill. 오미자

Taebaek-si(Hyeol-dong, Mt. Hambaek)-140605-035 [HNHM]

Lauraceae 녹나무과

90. Lindera obtusiloba Blume 생강나무

Jeongseon-gun(Gohan-ri, Mt. Hambaek)-140425-136 [HNHM]

Ranunculaceae 미나리아재비과

91. Aconitum ciliare DC. 놋젓가락나물

Taebaek-si(Changjuk-dong, Mt. Daedeok)-120920-250 [KB]

92. Aconitum jaluense Kom. 투구꽃

Jeongseon-gun(Gohan-ri, Mt. Hambaek)-140830-018 [HNHM]

$\star 93$. Aconitum koreanum R. Raymund 백부자

Taebaek-si(Changjuk-dong, Mt. Geumdae)-120815-047 [KB]

$\star$ 94. Aconitum macrorhynchum Turcz. 가는돌쩌귀

Jeongseon-gun(Gohan-ri, Mt. Hambaek)-140830-007 [HNHM]

95. Aconitum pseudolaeve Nakai 진범

Jeongseon-gun(Gohan-ri, Mt. Hambaek)-140830-014 [HNHM]

96. Actaea asiatica H. Hara 노루삼

Taebaek-si(Hyeol-dong, Mt. Hambaek)-140605-132 [HNHM]

$\star 97$. Adonis amurensis Regel \& Radde 복수초

Taebaek-si(Changjuk-dong, Mt. Daedeok)-120419-006 [KB]

98. Anemone koraiensis Nakai 홀아비바람꽃

Jeongseon-gun(Gohan-ri, Mt. Hambaek)-140425-123 [HNHM]

$\star 99$. Anemone raddeana Regel 꿩의바람꽃

Jeongseon-gun(Gohan-ri, Mt. Eundae)-140424-068 [HNHM]

$\star 100$. Anemone reflexa Steph. \& Willd. 회리바람꽃

Jeongseon-gun(Gohan-ri, Mt. Eundae)-140424-140 [HNHM]

$\star$ 101. Caltha palustris L. 동의나물

Jeongseon-gun(Gohan-ri, Mt. Hambaek)-140425-173 [HNHM]

$\star$ 102. Cimicifuga dahurica (Turcz. ex Fisch. \& C.A. Mey.) Maxim. 눈빛승마

Jeongseon-gun(Gohan-ri, Mt. Hambaek)-140830-015 [HNHM]

$\star$ 103. Cimicifuga foetida $\mathrm{L}$. 황새승마

Taebaek-si(Hwajeon-dong, Mt. Eundae)-140807-154 [HNHM]

104. Cimicifuga heracleifolia Kom. 승마

Jeongseon-gun(Gohan-ri, Mt. Hambaek)-120919-116 [KB]

105. Cimicifuga simplex (DC.) Turcz. 촛대승마

Jeongseon-gun(Gohan-ri, Mt. Hambaek)-140830-012 [HNHM] 
^106. Clematis fusca Turcz. 검종덩굴

Taebaek-si(Changjuk-dong, Mt. Geumdae)-120704-049 [KB]

107. Clematis fusca var. coreana (H. Lév. \& Vaniot) Nakai 요강나물 Jeongseon-gun(Gohan-ri, Mt. Hambaek)-130711-108 [KB]

$\star$ 108. Clematis fusca var. violacea Maxim. 종덩굴 Jeongseon-gun(Gohan-ri, Mt. Hambaek)-130828-261 [KB] 109. Clematis heracleifolia DC. 병조희풀 Taebaek-si(Hyeol-dong, Mt. Hambaek)-140704-059 [HNHM] 110. Clematis koreana Kom. 세잎종덩굴 Jeongseon-gun(Gohan-ri, Mt. Hambaek)-140522-087 [HNHM] 111. Clematis trichotoma Nakai 할미밀망 Taebaek-si(Hyeol-dong, Mt. Hambaek)-140605-051 [HNHM] $\star 112$. Enemion raddeanum Regel 나도바람꽃 Jeongseon-gun(Gohan-ri, Mt. Hambaek)-140425-101 [HNHM] $\star$ 113. Eranthis stellata Maxim. 너도바람꽃 Jeongseon-gun(Gohan-ri, Mt. Hambaek)-140425-188 [HNHM] $\star 114$. Hepatica asiatica Nakai 노루귀 Jeongseon-gun(Gohan-ri, Mt. Hambaek)-140425-023 [HNHM] 115. Pulsatilla koreana (Yabe ex Nakai) Nakai ex Mori 할미꽃 Taebaek-si(Changjuk-dong, Mt. Daedeok)-120525-198 [KB]

$\star 116$. Ranunculus chinensis Bunge 젓가락나물 Taebaek-si(Hyeol-dong, Mt. Hambaek)-140605-234 [HNHM] $\star$ 117. Ranunculus franchetii $\mathrm{H}$. Boissieu 왜미나리아재비 Jeongseon-gun(Gohan-ri, Mt. Hambaek)-130501-074 [KB] 118. Ranunculus japonicus Thunb. 미나리아재비 Jeongseon-gun(Baekjeon-ri, Mt. Daedeok)-140523-173 [HNHM]

$\star 119$. Ranunculus ternatus Thunb. 개구리갓

Jeongseon-gun(Gohan-ri, Mt. Eundae)-140424-119 [HNHM] 120. Thalictrum filamentosum var. tenerum (Huth) Ohwi 산꿩의다리 Taebaek-si(Hyeol-dong, Mt. Hambaek)-140704-130 [HNHM]

121. Thalictrum kemense var. hypoleucum (Siebold \& Zucc.) Kitag. 좀 뀡의다리

Taebaek-si(Changjuk-dong, Mt. Daedeok)-120920-310 [KB]

122. Thalictrum rochebrunianum var. grandisepalum (H. Lév.) Nakai 금꿩의다리

Samcheok-si(Hanso-ri, Mt. Daedeok)-130710-050 [KB]

Berberidaceae 매자나무과

123. Berberis amurensis Rupr. 매발톱나무

Jeongseon-gun(Gohan-ri, Mt. Hambaek)-140522-037 [HNHM]

124. Caulophyllum robustum Maxim. 꿩의다리아재비

Jeongseon-gun(Gohan-ri, Mt. Hambaek)-140522-115 [HNHM]

$\star 125$. Leontice microrrhyncha S. Moore 한계령풀

Jeongseon-gun(Gohan-ri, Mt. Hambaek)-130412-015 [KB]

Chloranthaceae 홀아비꽃대과

126. Chloranthus japonicus Siebold 홀아비꽃대

Taebaek-si(Hyeol-dong, Mt. Hambaek)-140605-130 [HNHM]

Aristolochiaceae 쥐방울덩굴과

127. Aristolochia manshuriensis Kom. 등칡

Taebaek-si(Hyeol-dong, Mt. Hambaek)-140704-152 [HNHM]

$\star 128$. Asarum heterotropoides var. seoulense (Nakai) Kitag. 서울족 도리풀

Jeongseon-gun(Gohan-ri, Mt. Hambaek)-130624-050 [KB]

129. Asarum sieboldii Miq. 족도리풀

Jeongseon-gun(Gohan-ri, Mt. Hambaek)-140425-132 [HNHM]

Actinidiaceae 다래나무과

130. Actinidia arguta (Siebold \& Zucc.) Planch. ex Miq. 다래 Taebaek-si(Hyeol-dong, Mt. Hambaek)-140704-184 [HNHM]

131. Actinidia kolomikta (Maxim. \& Rupr.) Maxim. 쥐다래 Taebaek-si(Hyeol-dong, Mt. Hambaek)-140605-190 [HNHM]

$\star$ 132. Actinidia polygama (Siebold \& Zucc.) Planch. ex Maxim. 개다래
Taebaek-si(Hyeol-dong, Mt. Hambaek)-140704-093 [HNHM] Guttiferae 물레나물과

133. Hypericum ascyron L. 물레나물

Taebaek-si(Hyeol-dong, Mt. Hambaek)-140705-102 [HNHM]

134. Hypericum erectum Thunb. 고추나물

Taebaek-si(Hwajeon-dong, Mt. Eundae)-140807-144 [HNHM]

Papaveraceae 양귀비과

$\star$ 135. Chelidonium majus var. asiaticum (Hara) Ohwi 애기똥풀 Taebaek-si(Hyeol-dong, Mt. Hambaek)-140605-067 [HNHM]

$\star 136$. Hylomecon vernalis Maxim. 피나물

Jeongseon-gun(Gohan-ri, Mt. Hambaek)-140425-180 [HNHM]

Fumariaceae 현호색과

$\star$ 137. Corydalis grandicalyx B.U. Oh \& Y.S. Kim 갈퀴현호색 Jeongseon-gun(Gohan-ri, Mt. Hambaek)-140425-018 [HNHM]

138. Corydalis pauciovulata Ohwi 선괴불주머니

Jeongseon-gun(Gohan-ri, Mt. Hambaek)-140425-040 [HNHM]

139. Corydalis remota Fisch. ex Maxim. 현호색

Jeongseon-gun(Gohan-ri, Mt. Hambaek)-130501-080 [KB]

140. Corydalis speciosa Maxim. 산괴불주머니

Jeongseon-gun(Gohan-ri, Mt. Hambaek)-130411-028 [KB]

Cruciferae 십자화과

141. Arabis gemmifera (Matsum.) Makino 산장대

Jeongseon-gun(Gohan-ri, Mt. Eundae)-140424-109 [HNHM]

142. Arabis glabra Bernh. 장대나물

Samcheok-si(Hanso-ri, Mt. Daedeok)-130710-057 [KB]

$\star 143$. Arabis lyrata L. 묏장대

Jeongseon-gun(Gohan-ri, Mt. Hambaek)-130828-170 [KB]

144. Arabis pendula L. 느러진장대

Jeongseon-gun(Gohan-ri, Mt. Hambaek)-130828-204 [KB]

145. Barbarea orthoceras Ledeb. 나도냉이

Jeongseon-gun(Baekjeon-ri, Mt. Daedeok)-140523-112 [HNHM]

$\star 146$. Berteroella maximowiczii (Palib.) O.E. Schulz 장대냉이

Taebaek-si(Hyeol-dong, Mt. Hambaek)-140605-059 [HNHM]

147. Capsella bursa-pastoris (L.) L.W. Medicus 냉이

Jeongseon-gun(Baekjeon-ri, Mt. Daedeok)-140523-159 [HNHM]

$\star 148$. Cardamine amaraeformis Nakai 꽃황새냉이

Jeongseon-gun(Gohan-ri, Mt. Hambaek)-140425-051 [HNHM]

149. Cardamine impatiens L. 싸리냉이

Jeongseon-gun(Gohan-ri, Mt. Hambaek)-140522-111 [HNHM]

150. Cardamine komarovi Nakai 는쟁이냉이

Jeongseon-gun(Gohan-ri, Mt. Hambaek)-120524-234 [KB]

151. Cardamine leucantha (Tausch) O.E. Schulz 미나리냉이

Taebaek-si(Hyeol-dong, Mt. Hambaek)-140704-227 [HNHM]

152. Draba nemorosa L. 꽃다지

Jeongseon-gun(Baekjeon-ri, Mt. Daedeok)-140523-061 [HNHM]

$\star$ 153. Rorippa indica (L.) Hiern 개갓냉이

Taebaek-si(Hyeol-dong, Mt. Hambaek)-140605-150 [HNHM]

154. Sisymbrium luteum (Maxim.) O.E. Schulz 노란장대

Taebaek-si(Hyeol-dong, Mt. Hambaek)-140605-208 [HNHM]

155. Thlaspi arvense L. 말냉이

Taebaek-si(Hyeol-dong, Mt. Hambaek)-140605-060 [HNHM]

\section{Crassulaceae 돌나물과}

156. Hylotelephium erythrostictum (Miq.) H. Ohba 뀡의비름 Jeongseon-gun(Gohan-ri, Mt. Hambaek)-131003-010 [KB] 157. Hylotelephium verticillatum (L.) H. Ohba 세잎뀡의비름 Jeongseon-gun(Gohan-ri, Mt. Hambaek)-130711-213 [KB]

$\star$ 158. Hylotelephium viviparum (Maxim.) H. Ohba 새끼꿩의비름 Taebaek-si(Hyeol-dong, Mt. Hambaek)-140705-140 [HNHM] 159. Sedum aizoon L. 가는기린초

Taebaek-si(Hwajeon-dong, Mt. Eundae)-140807-119 [HNHM] 
160. Sedum kamtschaticum Fisch. \& Mey. 기린초

Jeongseon-gun(Gohan-ri, Mt. Hambaek)-130624-003 [KB]

$\star$ 161. Sedum latiovalifolium Y.N. Lee 태백기린초 Jeongseon-gun(Gohan-ri, Mt. Hambaek)-130624-032 [KB] 162. Sedum polytrichoides Hemsl. 바위채송화 Taebaek-si(Hwajeon-dong, Mt. Eundae)-140807-238 [HNHM] 163. Sedum sarmentosum Bunge 돌나물 Taebaek-si(Hyeol-dong, Mt. Hambaek)-140705-123 [HNHM] Saxifragaceae 범의귀과

164. Astilbe rubra Hook. f. \& Thomas ex Hook. f. 노루오줌 Taebaek-si(Hwajeon-dong, Mt. Eundae)-140807-067 [HNHM]

$\star$ 165. Chrysosplenium flagelliferum $\mathrm{F}$. Schmidt 애기괭이눈 Taebaek-si(Hyeol-dong, Mt. Hambaek)-140704-103 [HNHM]

$\star$ 166. Chrysosplenium grayanum Maxim. 괭이눈

Jeongseon-gun(Gohan-ri, Mt. Hambaek)-140425-178 [HNHM]

$\star$ ^167. Chrysosplenium japonicum (Maxim.) Makino 산갱이눈 Jeongseon-gun(Gohan-ri, Mt. Hambaek)-140425-119 [HNHM]

$\star 168$. Chrysosplenium pilosum Maxim. 털갱이눈 Jeongseon-gun(Gohan-ri, Mt. Hambaek)-120703-113 [KB]

$\star 169$. Chrysosplenium pilosum var. fulvum (N. Terracc.) Hara 흰갱이눈 Jeongseon-gun(Gohan-ri, Mt. Hambaek)-120524-103 [KB]

$\star$ 170. Chrysosplenium pilosum var. sphaerospermum H. Hara 금괭이눈 Jeongseon-gun(Gohan-ri, Mt. Hambaek)-130501-151 [KB]

171. Chrysosplenium pseudofauriei $\mathrm{H}$. Lév. 선괭이눈 Jeongseon-gun(Gohan-ri, Mt. Hambaek)-130411-029 [KB]

$\star 172$. Chrysosplenium ramosum Maxim. 가지갱이눈 Jeongseon-gun(Gohan-ri, Mt. Hambaek)-120524-491 [KB] 173. Deutzia glabrata Kom. 물참대 Taebaek-si(Hyeol-dong, Mt. Hambaek)-140605-042 [HNHM]

$\star$ 174. Deutzia grandiflora var. baroniana Diels 바위말발도리 Jeongseon-gun(Gohan-ri, Mt. Hambaek)-120524-093 [KB]

$\star 175$. Deutzia parviflora Bunge 말발도리

Taebaek-si(Hyeol-dong, Mt. Hambaek)-140704-067 [HNHM] 176. Hydrangea serrata f. acuminata (Siebold \& Zucc.) Wilson 산수국 Taebaek-si(Hyeol-dong, Mt. Hambaek)-140704-336 [HNHM] $\star$ 177. Philadelphus schrenkii var. jackii Koehne 털고광나무 Samcheok-si(Panmun-ri, Mt. Daedeok)-120718-085 [KB]

$\star$ 178. Philadelphus tenuifolius Rupr. \& Maxim. 얇은잎고광나무 Taebaek-si(Hyeol-dong, Mt. Hambaek)-140605-183 [HNHM] 179. Ribes mandshuricum (Maxim.) Kom. 까치밥나무 Taebaek-si(Hyeol-dong, Mt. Hambaek)-140605-002 [HNHM] 180. Ribes maximowiczianum Kom. 명자순

Jeongseon-gun(Baekjeon-ri, Mt. Daedeok)-120814-138 [KB] 181. Saxifraga fortunei var. incisolobata (Engl. \& Irmsch.) Nakai 바위떡풀 Taebaek-si(Hyeol-dong, Mt. Hambaek)-140704-255 [HNHM]

$\star 182$. Saxifraga punctata L. 톱바위취

Jeongseon-gun(Gohan-ri, Mt. Hambaek)-130828-309 [KB]

^183. Saxifraga oblongifolia Nakai 참바위취

Jeongseon-gun(Gohan-ri, Mt. Hambaek)-140522-025 [HNHM]

Rosaceae 장미과

184. Agrimonia coreana Nakai 산짚신나물

Jeongseon-gun(Baekjeon-ri, Mt. Daedeok)-120814-095 [KB]

185. Agrimonia pilosa Ledeb. 짚신나물

Taebaek-si(Hwajeon-dong, Mt. Eundae)-140807-229 [HNHM]

$\star$ 186. Aruncus dioicus var. kamtschaticus (Maxim.) H. Hara 눈개승마

Jeongseon-gun(Gohan-ri, Mt. Hambaek)-130828-297 [KB]

$\star 187$. Crataegus pinnatifida Bunge 산사나무

Taebaek-si(Hyeol-dong, Mt. Hambaek)-140605-065 [HNHM]

188. Duchesnea indica (Andr.) Focke 뱀 딸기

Taebaek-si(Hyeol-dong, Mt. Hambaek)-140705-110 [HNHM]
189. Filipendula glaberrima (Nakai) Nakai 터리풀

Taebaek-si(Hyeol-dong, Mt. Hambaek)-140705-076 [HNHM]

$\star 190$. Filipendula koreana (Nakai) Nakai 붉은터리풀

Jeongseon-gun(Gohan-ri, Mt. Hambaek)-140522-104 [HNHM]

191. Geum aleppicum Jacq. 큰뱀무

Jeongseon-gun(Gohan-ri, Mt. Hambaek)-120703-165 [KB]

192. Geum japonicum Thunb. 뱀무

Taebaek-si(Hyeol-dong, Mt. Hambaek)-140705-154 [HNHM]

$\star$ 193. Kerria japonica f. pleniflora (Witte) Rehder 죽단화(식재)

Jeongseon-gun(Gohan-ri, Mt. Hambaek)-130828-118 [KB]

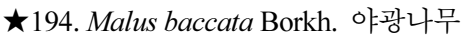

Jeongseon-gun(Baekjeon-ri, Mt. Daedeok)-140523-041 [HNHM]

$\star$ 195. Potentilla centigrana Maxim. 좀딸기

Taebaek-si(Hyeol-dong, Mt. Hambaek)-140704-205 [HNHM]

196. Potentilla chinensis Ser. 딱지꽃

Taebaek-si(Hyeol-dong, Mt. Hambaek)-140705-174 [HNHM]

197. Potentilla cryptotaeniae Maxim. 물양지꽃

Taebaek-si(Hyeol-dong, Mt. Hambaek)-140704-141 [HNHM]

198. Potentilla fragarioides var. major Maxim. 양지꽃

Jeongseon-gun(Gohan-ri, Mt. Eundae)-140424-132 [HNHM]

$\star$ 199. Prunus maackii Rupr. 개벚지나무

Jeongseon-gun(Baekjeon-ri, Mt. Daedeok)-140523-133 [HNHM]

$\star 200$. Prunus maximowiczii Rupr. 산개벚지나무

Taebaek-si(Hyeol-dong, Mt. Hambaek)-140605-205 [HNHM]

201. Prunus padus L. 귀룽나무

Jeongseon-gun(Gohan-ri, Mt. Hambaek)-140522-145 [HNHM]

$\star 202$. Prunus serrulata var. spontanea (Maxim.) E.H. Wilson 벚나무 Jeongseon-gun(Baekjeon-ri, Mt. Daedeok)-140523-116 [HNHM] 203. Pyrus ussuriensis Maxim. 산돌배

Taebaek-si(Hyeol-dong, Mt. Hambaek)-140704-221 [HNHM]

204. Rosa davurica Pall. 생열귀나무

Jeongseon-gun(Gohan-ri, Mt. Hambaek)-140522-173 [HNHM]

205. Rosa davurica var. ellipsoidea Nakai 긴생열귀

Jeongseon-gun(Gohan-ri, Mt. Hambaek)-130828-314 [KB]

206. Rosa multiflora Thunb. 찔레나무

Taebaek-si(Hwajeon-dong, Mt. Eundae)-140807-225 [HNHM]

$\star 207$. Rosa suavis Willd. 인가목

Jeongseon-gun(Gohan-ri, Mt. Hambaek)-120705-077 [KB]

208. Rubus crataegifolius Bunge 산딸기

Jeongseon-gun(Gohan-ri, Mt. Hambaek)-120705-067 [HNHM]

$\star 209$. Rubus idaeus var. microphyllus Turcz. 멍덕딸기

Jeongseon-gun(Gohan-ri, Mt. Hambaek)-090708-70314 [HNHM]

210. Rubus oldhamii Miq. 줄딸기

Jeongseon-gun(Gohan-ri, Mt. Hambaek)-140522-119 [HNHM]

211. Rubus phoenicolasius Maxim. 곰딸기

Taebaek-si(Hyeol-dong, Mt. Hambaek)-140704-046 [HNHM]

212. Sanguisorba officinalis L. 오이풀

Taebaek-si(Hwajeon-dong, Mt. Eundae)-140807-203 [HNHM]

213. Sorbaria sorbifolia var. stellipila Maxim. 쉬땅나무

Taebaek-si(Hwajeon-dong, Mt. Eundae)-140807-156 [HNHM]

214. Sorbus alnifolia (Siebold \& Zucc.) K.Koch 팥배나무 Jeongseon-gun(Gohan-ri, Mt. Hambaek)-130711-227 [KB]

215. Sorbus amurensis Koehne 당마가목

Taebaek-si(Hyeol-dong, Mt. Hambaek)-140704-322 [HNHM]

216. Sorbus commixta Hedlund 마가목

Jeongseon-gun(Gohan-ri, Mt. Hambaek)-140522-103 [HNHM]

\217. Spiraea blumei G.Don 산조팝나무

Taebaek-si(Hyeol-dong, Mt. Hambaek)-140705-086 [HNHM]

218. Spiraea chamaedryfolia L. 인가목조팝나무

Taebaek-si(Changjuk-dong, Mt. Daedeok)-120525-217 [KB] 
^219. Spiraea fritschiana Schneid. 참조팝나무

Jeongseon-gun(Gohan-ri, Mt. Hambaek)-130828-286 [KB]

$\star 220$. Spiraea miyabei Koidz. 덤불조팝나무

Jeongseon-gun(Gohan-ri, Mt. Hambaek)-130711-201 [KB]

221. Spiraea prunifolia f. simpliciflora Nakai 조팝나무

Jeongseon-gun(Gohan-ri, Mt. Hambaek)-120524-385 [KB]

222. Spiraea pubescens Turcz. 아구장나무

Taebaek-si(Changjuk-dong, Mt. Daedeok)-120920-009 [KB]

223. Spiraea trichocarpa Nakai 갈기조팝나무

Samcheok-si(Hanso-ri, Mt. Daedeok)-130710-042 [KB]

224. Stephanandra incisa (Thunb.) Zabel 국수나무

Taebaek-si(Hyeol-dong, Mt. Hambaek)-140705-146 [HNHM]

225. Waldsteinia ternata (Stephan) Fritsch 나도양지꽃

Jeongseon-gun(Gohan-ri, Mt. Hambaek)-140425-155 [HNHM]

Leguminosae 콩과

\226. Amorpha fruticosa L. 족제비싸리

Taebaek-si(Hwajeon-dong, Mt. Eundae)-140807-013 [HNHM]

$\star 227$. Amphicarpaea bracteata subsp. edgeworthii (Benth.) H. Ohashi 새콩

Jeongseon-gun(Gohan-ri, Mt. Hambaek)-131003-146 [KB]

$\star 228$. Astragalus sinicus L. 자운영

Taebaek-si(Hyeol-dong, Mt. Hambaek)-140705-118 [HNHM]

229. Desmodium podocarpum DC. 개도둑놈의갈고리

Samcheok-si(Panmun-ri, Mt. Daedeok)-120718-025 [KB]

230. Desmodium podocarpum var. oxyphyllum (DC.) H. Ohashi 도둑 놈의갈고리

Taebaek-si(Hyeol-dong, Mt. Hambaek)-140704-074 [HNHM]

$\star 231$. Glycine soja Siebold \& Zucc. 돌콩

Taebaek-si(Hwajeon-dong, Mt. Eundae)-140807-057 [HNHM]

$\star 232$. Kummerowia striata (Thunb.) Schindl. 매듭풀

Taebaek-si(Hwajeon-dong, Mt. Eundae)-140807-110 [HNHM]

233. Lathyrus davidii Hance 활량나물

Samcheok-si(Hanso-ri, Mt. Daedeok)-130710-126 [KB]

234. Lespedeza bicolor Turcz. 싸리

Samcheok-si(Hanso-ri, Mt. Daedeok)-130710-135 [KB]

$\star 235$. Lespedeza cuneata G. Don 비수리

Taebaek-si(Hwajeon-dong, Mt. Eundae)-140807-185 [HNHM]

236. Lespedeza cyrtobotrya Miq. 참싸리

Taebaek-si(Hyeol-dong, Mt. Hambaek)-140705-145 [HNHM]

$\star 237$. Lespedeza maximowiczii C.K. Schneid. 조록싸리

Taebaek-si(Hyeol-dong, Mt. Hambaek)-140705-002 [HNHM]

$\star 238$. Lotus corniculatus var. japonica Regel 벌노랑이

Taebaek-si(Hwajeon-dong, Mt. Eundae)-140807-128 [HNHM]

239. Maackia amurensis Rupr. \& Maxim. 다릅나무

Taebaek-si(Hyeol-dong, Mt. Hambaek)-140704-237 [HNHM]

$\star 240$. Medicago lupulina L. 잔개자리

Taebaek-si(Hyeol-dong, Mt. Hambaek)-140705-172 [HNHM]

$\star 241$. Medicago polymorpha L. 개자리

Jeongseon-gun(Gohan-ri, Mt. Hambaek)-120703-008 [KB]

$\star 242$. Melilotus alba Medicus 흰전동싸리

Taebaek-si(Hyeol-dong, Mt. Hambaek)-140705-063 [HNHM]

243. Melilotus suaveolens Ledeb. 전동싸리

Taebaek-si(Hwajeon-dong, Mt. Eundae)-140807-031 [HNHM]

$\star 244$. Pueraria lobata (Willd.) Ohwi 칡

Taebaek-si(Hwajeon-dong, Mt. Eundae)-140807-241 [HNHM]

245. Trifolium pratense L. 붉은토끼풀

Taebaek-si(Hyeol-dong, Mt. Hambaek)-140705-170 [HNHM]

246. Trifolium repens L. 토끼풀

Samcheok-si(Hanso-ri, Mt. Daedeok)-130710-281 [KB]

$\star 247$. Vicia amoena Fisch. ex DC. 갈퀴나물
Samcheok-si(Panmun-ri, Mt. Daedeok)-120718-004 [KB]

248. Vicia amurensis Oett. 벌완두

Samcheok-si(Panmun-ri, Mt. Daedeok)-120718-240 [KB]

$\star 249$. Vicia chosenensis Ohwi 노랑갈퀴

Taebaek-si(Hyeol-dong, Mt. Hambaek)-140605-089 [HNHM]

$\star 250$. Vicia cracca L. 등갈퀴나물

Taebaek-si(Hwajeon-dong, Mt. Eundae)-140807-111 [HNHM]

251. Vicia unijuga A. Braun 나비나물

Jeongseon-gun(Gohan-ri, Mt. Hambaek)-131003-153 [KB]

252. Vicia venosa var. cuspidata Maxim. 광릉갈퀴

Jeongseon-gun(Gohan-ri, Mt. Hambaek)-130828-127 [KB]

Oxalidaceae 갱이밥과

$\star 253$. Oxalis corniculata L. 괭이밥

Jeongseon-gun(Gohan-ri, Mt. Hambaek)-120524-105 [KB]

$\star 254$. Oxalis obtriangulata Maxim. 큰갱이밥

Jeongseon-gun(Gohan-ri, Mt. Hambaek)-140425-089 [HNHM]

Geraniaceae 쥐손이풀과

\255. Geranium knuthii Nakai 큰세잎쥐손이

Jeongseon-gun(Gohan-ri, Mt. Hambaek)-130828-293 [KB]

$\star 256$. Geranium koreanum Kom. 둥근이질풀

Jeongseon-gun(Gohan-ri, Mt. Hambaek)-130828-092 [KB]

$\star 257$. Geranium koreanum var. hirsutum Nakai 털둥근이질풀

Jeongseon-gun(Gohan-ri, Mt. Eundae)-120704-031 [KH]

$\star 258$. Geranium krameri Franch. \& Sav. 선이질풀

Jeongseon-gun(Gohan-ri, Mt. Hambaek)-090708-69722 [HNHM]

259. Geranium sibiricum L. 쥐손이풀

Taebaek-si(Hwajeon-dong, Mt. Eundae)-140807-007 [HNHM]

$\star 260$. Geranium taebaek S.J. Park \& Y.S. Kim 태백이질풀

Jeongseon-gun(Gohan-ri, Mt. Hambaek)-130624-042 [KB]

$\star 261$. Geranium wilfordii Maxim. 세잎쥐손이

Jeongseon-gun(Gohan-ri, Mt. Hambaek)-130711-097 [KB]

Euphorbiaceae 대극과

262. Acalypha australis L. 깨풀

Jeongseon-gun(Baekjeon-ri, Mt. Daedeok)-120814-180 [KB]

263. Euphorbia sieboldiana Morren \& Decne. 개감수

Taebaek-si(Hyeol-dong, Mt. Hambaek)-140605-152 [HNHM]

$\star 264$. Securinega suffruticosa (Pall.) Rehder 광대싸리

Taebaek-si(Hyeol-dong, Mt. Hambaek)-140705-258 [HNHM]

Rutaceae 운향과

265. Phellodendron amurense Rupr. 황벽나무

Jeongseon-gun(Baekjeon-ri, Mt. Daedeok)-140523-152 [HNHM]

Anacardiaceae 옻나무과

266. Rhus javanica L. 붉나무

Taebaek-si(Hwajeon-dong, Mt. Eundae)-140807-168 [HNHM]

267. Rhus tricocarpa Miq. 개옻나무

Jeongseon-gun(Gohan-ri, Mt. Hambaek)-120703-134 [KB]

Aceraceae 단풍나무과

268. Acer komarovii Pojark. 시닥나무

Taebaek-si(Hyeol-dong, Mt. Hambaek)-140605-081 [HNHM]

269. Acer mandshuricum Maxim. 복장나무

Jeongseon-gun(Gohan-ri, Mt. Hambaek)-120524-292 [KB]

$\star 270$. Acer pictum Thunb. 털고로쇠나무

Samcheok-si(Panmun-ri, Mt. Daedeok)-120718-054 [KB]

271. Acer pictum subsp. mono (Maxim.) Ohashi 고로쇠나무

Taebaek-si(Hyeol-dong, Mt. Hambaek)-140704-216 [HNHM]

272. Acer pseudosieboldianum (Pax) Kom. 당단풍나무

Jeongseon-gun(Gohan-ri, Mt. Hambaek)-120524-473 [KB]

$\star 273$. Acer tataricum subsp. ginnala (Maxim.) Wesm. 신나무

Taebaek-si(Hyeol-dong, Mt. Hambaek)-140704-087 [HNHM]

274. Acer tegmentosum Maxim. 산겨릅나무 
Jeongseon-gun(Baekjeon-ri, Mt. Daedeok)-140523-262 [HNHM] 275. Acer ukurunduense Trautv. \& C.A. Mey. 부게꽃나무 Jeongseon-gun(Gohan-ri, Mt. Hambaek)-140522-036 [HNHM] Balsaminaceae 봉선화과

276. Impatiens noli-tangere L. 노랑물봉선

Jeongseon-gun(Gohan-ri, Mt. Hambaek)-130711-195 [KB]

$\star 277$. Impatiens textori Miq. 물봉선

Taebaek-si(Hwajeon-dong, Mt. Eundae)-140807-104 [HNHM]

$\star 278$. Impatiens textori var. koreana Nakai 흰물봉선

Taebaek-si(Hwajeon-dong, Mt. Eundae)-140807-196 [HNHM] Celastraceae 노박덩굴과

279. Celastrus orbiculatus Thunb. 노박덩굴

Taebaek-si(Hwajeon-dong, Mt. Eundae)-140807-171 [HNHM]

280. Euonymus alatus (Thunb.) Siebold 화살나무

Taebaek-si(Hyeol-dong, Mt. Hambaek)-140605-053 [HNHM]

281. Euonymus alatus f. ciliatodentatus (Franch. \& Sav.) Hiyama 회잎나무 Jeongseon-gun(Baekjeon-ri, Mt. Daedeok)-140523-029 [HNHM] 282. Euonymus hamiltonianus Wall. 참빗살나무 Jeongseon-gun(Gohan-ri, Mt. Hambaek)-130711-196 [KB] 283. Euonymus macropterus Rupr. 나래회나무 Jeongseon-gun(Gohan-ri, Mt. Hambaek)-130711-085 [KB]

$\star 284$. Euonymus oxyphyllus Miq. 참회나무

Jeongseon-gun(Gohan-ri, Mt. Hambaek)-140425-014 [HNHM]

285. Euonymus pauciflorus Maxim. 회목나무

Jeongseon-gun(Gohan-ri, Mt. Hambaek)-120703-109 [KB]

286. Euonymus sachalinensis (F. Schmidt) Maxim. 회나무

Taebaek-si(Hyeol-dong, Mt. Hambaek)-140705-070 [HNHM]

287. Tripterygium regelii Sprague \& Takeda 미역줄나무

Taebaek-si(Hwajeon-dong, Mt. Eundae)-140807-235 [HNHM]

Staphyleaceae 고추나무과

288. Staphylea bumalda DC. 고추나무

Taebaek-si(Hyeol-dong, Mt. Hambaek)-140704-142 [HNHM]

Rhamnaceae 갈매나무과

$\star 289$. Rhamnus davurica Pall. 갈매나무

Jeongseon-gun(Gohan-ri, Mt. Hambaek)-140522-029 [HNHM]

$\star 290$. Rhamnus koraiensis C.K. Schneid. 털갈매나무

Jeongseon-gun(Gohan-ri, Mt. Hambaek)-130828-247 [KB]

291. Rhamnus yoshinoi Makino 짝자래나무

Taebaek-si(Hyeol-dong, Mt. Hambaek)-140704-137 [HNHM]

Vitaceae 포도과

$\star 292$. Ampelopsis brevipedunculata (Maxim.) Trautv. 개머루 Jeongseon-gun(Gohan-ri, Mt. Hambaek)-120524-071 [KB]

$\star 293$. Vitis amurensis Rupr. 왕머루

Taebaek-si(Hyeol-dong, Mt. Hambaek)-140605-179 [HNHM] Tiliaceae 피나무과

294. Corchoropsis tomentosa (Thunb.) Makino 수까치깨 Jeongseon-gun(Baekjeon-ri, Mt. Daedeok)-120814-147 [KB]

295. Tilia amurensis Rupr. 피나무

Taebaek-si(Hyeol-dong, Mt. Hambaek)-140705-044 [HNHM]

^296. Tilia taquetii C.K. Schneid. 뽕잎피나무

Taebaek-si(Changjuk-dong, Mt. Geumdae)-120704-088 [KB]

Elaeagnaceae 보리수나무과

\297. Elaeagnus macrophylla Thunb. 보리밥나무

Taebaek-si(Hwajeon-dong, Mt. Eundae)-140807-112 [HNHM]

298. Elaeagnus umbellata Thunb. 보리수나무

Taebaek-si(Hyeol-dong, Mt. Hambaek)-140605-142 [HNHM]

Violaceae 제비꽃과

299. Viola acuminata Ledeb. 졸방제비꽃

Jeongseon-gun(Baekjeon-ri, Mt. Daedeok)-140523-191 [HNHM] 300. Viola albida Palib. 태백제비꽃
Jeongseon-gun(Gohan-ri, Mt. Hambaek)-140425-041 [HNHM] 301. Viola albida f. takahashii (Makino) W.T. Lee 단풍제비꽃 Taebaek-si(Hyeol-dong, Mt. Hambaek)-140605-025 [HNHM] 302. Viola albida var. chaerophylloides (Regel) F. Maek. 남산제비꽃 Taebaek-si(Hyeol-dong, Mt. Hambaek)-140605-193 [HNHM] 303. Viola collina Besser 둥근털제비꽃

Jeongseon-gun(Gohan-ri, Mt. Eundae)-140424-126 [HNHM]

$\star 304$. Viola diamantiaca Nakai 금강제비꽃

Jeongseon-gun(Gohan-ri, Mt. Hambaek)-140425-107 [HNHM]

$\star 305$. Viola japonica Langsdorf ex Ging. 왜제비꽃

Jeongseon-gun(Gohan-ri, Mt. Hambaek)-140425-190 [HNHM]

$\star$ 306. Viola keiskei Miq. 잔털제비꽃

Taebaek-si(Hyeol-dong, Mt. Hambaek)-140704-150 [HNHM]

307. Viola mandshurica W. Becker 제비꽃

Taebaek-si(Changjuk-dong, Mt. Daedeok)-120525-180 [KB]

308. Viola orientalis (Maxim.) W. Becker 노랑제비꽃

Jeongseon-gun(Gohan-ri, Mt. Eundae)-140424-098 [HNHM]

309. Viola phalacrocarpa Maxim. 털제비꽃

Jeongseon-gun(Gohan-ri, Mt. Eundae)-140424-131 [HNHM]

310. Viola selkirkii Pursh ex Goldie 뫼제비꽃

Jeongseon-gun(Gohan-ri, Mt. Hambaek)-120524-076 [KB]

$\star 311$. Viola tokubuchiana var. takedana (Makino) F. Maek. 민둥뫼 제비꽃

Jeongseon-gun(Gohan-ri, Mt. Hambaek)-130501-119 [HNHM]

312. Viola verecunda A. Gray 콩제비꽃

Taebaek-si(Hwajeon-dong, Mt. Eundae)-140807-083 [HNHM]

$\star 313$. Viola yedoensis Makino 호제비꽃

Jeongseon-gun(Gohan-ri, Mt. Hambaek)-140522-158 [HNHM]

Cucurbitaceae 박과

314. Schizopepon bryoniaefolius Maxim. 산외

Taebaek-si(Hyeol-dong, Mt. Hambaek)-140704-012 [HNHM]

Onagraceae 바늘꽃과

$\star 315$. Circaea alpina L. 쥐털이슬

Jeongseon-gun(Gohan-ri, Mt. Hambaek)-130828-342 [KB]

$\star$ 316. Circaea mollis Slebold \& Zucc. 털이슬

Taebaek-si(Hwajeon-dong, Mt. Eundae)-140807-048 [HNHM]

317. Epilobium cephalostigma Hausskn. 돌바늘꽃

Jeongseon-gun(Baekjeon-ri, Mt. Daedeok)-120814-004 [KB]

318. Epilobium pyrricholophum Franch. \& Sav. 바늘꽃

Taebaek-si(Hwajeon-dong, Mt. Eundae)-140807-079 [HNHM]

319. Oenothera biennis L. 달맞이꽃

Samcheok-si(Panmun-ri, Mt. Daedeok)-120718-107 [KB]

Alangiaceae 박쥐나무과

320. Alangium platanifolium var. trilobum (Miq.) Ohwi 박쥐나무

Taebaek-si(Hyeol-dong, Mt. Hambaek)-140704-170 [HNHM]

Cornaceae 충충나무과

321. Cornus controversa Hemsl. ex Prain 층층나무

Taebaek-si(Hyeol-dong, Mt. Hambaek)-140605-143 [HNHM]

$\star 322$. Cornus macrophylla Wall. 곰의말채나무

Taebaek-si(Hyeol-dong, Mt. Hambaek)-140705-073 [HNHM]

Araliaceae 두릅나무과

323. Aralia cordata var. continentalis (Kitag.) Y.C. Chu 독활

Taebaek-si(Hyeol-dong, Mt. Hambaek)-140705-124 [HNHM]

324. Aralia elata (Miq.) Seem. 두릅나무

Taebaek-si(Hwajeon-dong, Mt. Eundae)-140807-222 [HNHM]

$\star$ 325. Eleutherococcus senticosus (Rupr. \& Maxim.) Maxim. 가시오갈피 Taebaek-si(Hyeol-dong, Mt. Hambaek)-140704-335 [HNHM]

326. Eleutherococcus sessiliflorus (Rupr. \& Maxim.) S.Y. Hu 오갈피나무 Taebaek-si(Hyeol-dong, Mt. Hambaek)-140704-407 [HNHM] 327. Kalopanax septemlobus (Thunb.) Koidz. 음나무 
Jeongseon-gun(Baekjeon-ri, Mt. Daedeok)-140523-261 [HNHM] Umbelliferae 산형과

$\star 328$. Angelica acutiloba (Siebold \& Zucc.) Kitag. 왜당귀 Jeongseon-gun(Gohan-ri, Mt. Hambaek)-130711-133 [KB]

$\star 329$. Angelica anomala Ave-Lall. 개구릿대

Jeongseon-gun(Gohan-ri, Mt. Hambaek)-130828-120 [KB]

330. Angelica czernaevia (Fisch. \& C.A. Mey.) Kitag. 잔잎바디 Taebaek-si(Changjuk-dong, Mt. Daedeok)-120920-197 [KB]

331. Angelica dahurica (Fisch. ex Hoffm) Benth. \& Hook. f. ex Franch.\& Sav. 구릿대

Taebaek-si(Hwajeon-dong, Mt. Eundae)-140807-024 [HNHM]

332. Angelica decursiva (Miq.) Franch. \& Sav. 바디나물 Jeongseon-gun(Gohan-ri, Mt. Hambaek)-131003-154 [KB]

$\star$ 333. Angelica genuflexa Nutt. ex Torrey \& A. Gray 왜천궁 Jeongseon-gun(Gohan-ri, Mt. Eundae)-120704-007 [KB] 334. Angelica gigas Nakai 참당귀 Jeongseon-gun(Gohan-ri, Mt. Hambaek)-130828-335 [KB] 335. Angelica polymorpha Maxim. 궁궁이 Jeongseon-gun(Gohan-ri, Mt. Hambaek)-130828-084 [KB] 336. Angelica tenuissima Nakai 고본 Jeongseon-gun(Gohan-ri, Mt. Hambaek)-131003-087 [KB] 337. Anthriscus sylvestris (L.) Hoffm. 전호 Taebaek-si(Hyeol-dong, Mt. Hambaek)-140605-188 [HNHM] $\star$ 338. Bupleurum longeradiatum Turcz. 개시호 Jeongseon-gun(Gohan-ri, Mt. Hambaek)-130828-179 [KB] $\star$ 339. Cicuta virosa L. 독미나리 Jeongseon-gun(Gohan-ri, Mt. Hambaek)-120705-039 [KB] 340. Cryptotaenia japonica Hassk. 파드득나물 Taebaek-si(Changjuk-dong, Mt. Daedeok)-120920-020 [KB]

$\star 341$. Cymopterus melanotilingia (H. Boissieu) C.Y. Yoon 큰참나물 Jeongseon-gun(Gohan-ri, Mt. Hambaek)-130828-262 [KB] 342. Heracleum moellendorffii Hance 어수리 Jeongseon-gun(Gohan-ri, Mt. Hambaek)-131003-091 [KB] $\star 343$. Heracleum moellendorffii f. angustum (Kitag.) Kitag. 좁은어수리 Jeongseon-gun(Gohan-ri, Mt. Hambaek)-131003-068 [KB] 344. Osmorhiza aristata (Thunb.) Makino \& Yabe 긴사상자 Samcheok-si(Hanso-ri, Mt. Daedeok)-130710-194 [KB] 345. Ostericum grosseserratum (Maxim.) Kitag. 신감채 Taebaek-si(Hwajeon-dong, Mt. Eundae)-140807-198 [HNHM]

$\star 346$. Ostericum praeteritum Kitag. 강활

Jeongseon-gun(Gohan-ri, Mt. Hambaek)-130828-200 [KB]

347. Ostericum sieboldii (Miq.) Nakai 묏미나리

Jeongseon-gun(Gohan-ri, Mt. Hambaek)-130828-226 [KB] 348. Peucedanum terebinthaceum (Fisch.) Fisch. ex DC. 기름나물 Taebaek-si(Hwajeon-dong, Mt. Eundae)-140807-208 [HNHM] 349. Pimpinella brachycarpa (Kom.) Nakai 참나물 Jeongseon-gun(Gohan-ri, Mt. Hambaek)-130828-091 [KB]

$\star 350$. Pimpinella koreana (Yabe) Nakai 가는참나물 Jeongseon-gun(Gohan-ri, Mt. Hambaek)-131003-094 [KB]

$\star 351$. Sanicula chinensis Bunge 참반디

Taebaek-si(Hyeol-dong, Mt. Hambaek)-140704-011 [HNHM] 352. Sanicula rubriflora F. Schmidt ex Maxim. 붉은참반디 Taebaek-si(Changjuk-dong, Mt. Geumdae)-120704-095 [KB] $\star 353$. Sphallerocarpus gracilis (Besser) Koso-Pol. 무산상자 Taebaek-si(Hyeol-dong, Mt. Hambaek)-140704-125 [HNHM] 354. Torilis japonica (Houtt.) DC. 사상자 Jeongseon-gun(Baekjeon-ri, Mt. Daedeok)-140523-026 [HNHM] $\star 355$. Torilis scabra (Thunb.) DC. 개사상자 Taebaek-si(Hyeol-dong, Mt. Hambaek)-140704-185 [HNHM] Pyrolaceae 노루발과 $\star$ 356. Pyrola japonica Klenze ex Alef. 노루발

Taebaek-si(Hwajeon-dong, Mt. Eundae)-140807-121 [HNHM]

\section{Ericaceae 진달래과}

357. Rhododendron mucronulatum Turcz. 진달래

Taebaek-si(Hyeol-dong, Mt. Hambaek)-140705-238 [HNHM]

358. Rhododendron schlippenbachii Maxim. 철쭉

Jeongseon-gun(Gohan-ri, Mt. Hambaek)-140522-127 [HNHM]

359. Rhododendron yedoense f. poukhanense (H. Lév.) Sugim. 산철쭉 Jeongseon-gun(Gohan-ri, Mt. Hambaek)-120524-107 [KB]

360. Vaccinium hirtum var. koreanum (Nakai) Kitam. 산앵도나무 Jeongseon-gun(Gohan-ri, Mt. Hambaek)-130828-185 [KB]

$\star 361$. Vaccinium oldhamii Miq. 정금나무

Jeongseon-gun(Gohan-ri, Mt. Hambaek)-130828-010 [KB]

Primulaceae 앵초과

362. Lysimachia clethroides Duby 큰까치수염

Taebaek-si(Hyeol-dong, Mt. Hambaek)-140705-113 [HNHM]

363. Lysimachia vulgaris var. davurica (Ledeb.) R. Kunth 좁쌀풀 Jeongseon-gun(Gohan-ri, Mt. Hambaek)-130711-039 [KB]

$\star 364$. Primula jesoana Miq. 큰앵초

Jeongseon-gun(Gohan-ri, Mt. Hambaek)-120703-116 [KB]

$\star$ 365. Trientalis europaea var. arctica (Fisch.) Ledeb. 기생꽃

Jeongseon-gun(Gohan-ri, Mt. Hambaek)-140522-085 [HNHM]

Symplocaceae 노린재나무과

366. Symplocos chinensis f. pilosa (Nakai) Ohwi 노린재나무

Samcheok-si(Hanso-ri, Mt. Daedeok)-130710-191 [KB]

\section{Oleaceae 물푸레나무과}

367. Fraxinus rhynchophylla Hance 물푸레나무

Taebaek-si(Hyeol-dong, Mt. Hambaek)-140605-229 [HNHM]

368. Fraxinus sieboldiana Blume 쇠물푸레나무

Jeongseon-gun(Gohan-ri, Mt. Hambaek)-120524-188 [KB]

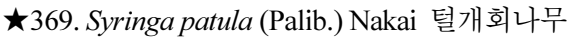

Jeongseon-gun(Gohan-ri, Mt. Hambaek)-120524-231 [KB]

370. Syringa reticulata var. mandshurica (Maxim.) H. Hara 개회나무 Taebaek-si(Hyeol-dong, Mt. Hambaek)-140605-120 [HNHM]

371. Syringa wolfii C.K.Schneid. 꽃개회나무

Jeongseon-gun(Gohan-ri, Mt. Hambaek)-120705-044 [KH]

Gentianaceae 용담과

372. Anagallidium dichotomum (L.) Grisb. 대성쓴풀

Taebaek-si(Changjuk-dong, Mt. Daedeok)-120525-357 [KB]

$\star 373$. Gentiana scabra Bunge 용담

Jeongseon-gun(Gohan-ri, Mt. Hambaek)-131003-110 [KB]

374. Gentiana squarrosa Ledeb. 구슬붕이

Jeongseon-gun(Baekjeon-ri, Mt. Daedeok)-140523-127 [HNHM]

375. Gentiana zollingeri Faw. 큰구슬붕이

Jeongseon-gun(Gohan-ri, Mt. Hambaek)-140522-156 [HNHM]

376. Swertia pseudochinensis H. Hara 자주쓴풀

Taebaek-si(Changjuk-dong, Mt. Daedeok)-120920-229 [KB]

Asclepiadaceae 박주가리과

$\star$ 377. Cynanchum ascyrifolium (Franch \& Sav.) Matsum. 민백미꽃 Taebaek-si(Hyeol-dong, Mt. Hambaek)-140705-033 [HNHM]

378. Cynanchum atratum Bunge 백미꽃

Taebaek-si(Changjuk-dong, Mt. Daedeok)-120525-282 [KB]

379. Cynanchum inamoenum (Maxim.) Loes. 선백미꽃

Taebaek-si(Changjuk-dong, Mt. Daedeok)-120920-145 [KB]

$\star$ 380. Metaplexis japonica (Thunb.) Makino 박주가리

Taebaek-si(Hwajeon-dong, Mt. Eundae)-140807-226 [HNHM]

Rubiaceae 꼭두서니과

$\star 381$. Asperula maximowiczii Kom. 개갈퀴

Taebaek-si(Hyeol-dong, Mt. Hambaek)-140704-260 [HNHM]

$\star 382$. Asperula odorata L. 선갈퀴 
Jeongseon-gun(Baekjeon-ri, Mt. Daedeok)-140523-219 [HNHM]

$\star 383$. Galium dahuricum Turcz. 큰잎갈퀴

Jeongseon-gun(Gohan-ri, Mt. Hambaek)-130828-098 [KB]

$\star$ 384. Galium dahuricum var. tokyoense (Makino) Cufodo. 흰갈퀴

Taebaek-si(Hyeol-dong, Mt. Hambaek)-140704-189 [HNHM]

$\star 385$. Galium paradoxum Maxim. 두메갈퀴

Taebaek-si(Hyeol-dong, Mt. Hambaek)-140605-173 [HNHM]

$\star 386$. Galium spurium var. echinospermum (Wallr.) Hayek 갈퀴덩굴

Samcheok-si(Hanso-ri, Mt. Daedeok)-130710-256 [KB]

387. Galium verum var. asiaticum Nakai 솔나물

Samcheok-si(Hanso-ri, Mt. Daedeok)-130710-232 [KB]

$\star 388$. Rubia akane Nakai 꼭두서니

Taebaek-si(Hwajeon-dong, Mt. Eundae)-140807-218 [HNHM]

389. Rubia chinensis Regel \& Maack 큰꼭두서니

Taebaek-si(Hyeol-dong, Mt. Hambaek)-140704-169 [HNHM]

390. Rubia cordifolia var. pratensis Maxim. 갈퀴꼭두서니

Taebaek-si(Hwajeon-dong, Mt. Eundae)-140807-216 [HNHM]

Convolvulaceae 메꽃과

391. Cuscuta japonica Choisy 새삼

Taebaek-si(Changjuk-dong, Mt. Daedeok)-120920-035 [KB]

Boraginaceae 지치과

392. Brachybotrys paridiformis Maxim. ex D. Oliver 당개지치

Taebaek-si(Hyeol-dong, Mt. Hambaek)-140605-094 [HNHM]

$\star$ 393. Symphytum officinale L. 컴프리

Taebaek-si(Hyeol-dong, Mt. Hambaek)-140705-234 [HNHM]

394. Trigonotis radicans var. sericea (Maxim.) H. Hara 참꽃마리

Taebaek-si(Changjuk-dong, Mt. Daedeok)-120525-140 [KB]

Labiatae 꿀풀과

395. Agastache rugosa (Fisch. \& Mey.) Kuntze 배초향

Taebaek-si(Hwajeon-dong, Mt. Eundae)-140807-223 [HNHM]

396. Clinopodium chinense var. parviflorum (Kudô) Hara 층층이꽃

Taebaek-si(Hwajeon-dong, Mt. Eundae)-140807-027 [HNHM]

397. Clinopodium micranthum (Regel) Hara 두메층층이

Taebaek-si(Hwajeon-dong, Mt. Eundae)-140807-097 [HNHM]

398. Elsholtzia ciliata (Thunb.) Hyl. 향유

Jeongseon-gun(Gohan-ri, Mt. Hambaek)-120919-054 [KB]

399. Elsholtzia splendens Nakai 꽃향유

Jeongseon-gun(Gohan-ri, Mt. Hambaek)-120705-003 [KB]

400. Isodon excisus (Maxim.) Kudô 오리방풀

Taebaek-si(Hwajeon-dong, Mt. Eundae)-140807-152 [HNHM]

401. Isodon inflexus (Thunb.) Kudô 산박하

Taebaek-si(Hwajeon-dong, Mt. Eundae)-140807-078 [HNHM]

402. Isodon japonicus (Burm.) Hara 방아풀

Taebaek-si(Changjuk-dong, Mt. Daedeok)-120920-194 [KB]

$\star 403$. Isodon serra (Maxim.) Kudô 자주방아풀

Taebaek-si(Changjuk-dong, Mt. Daedeok)-120920-030 [KB]

404. Lamium album var. barbatum (Siebold \& Zucc.) Franch. \& Sav. 광대수염

Taebaek-si(Hyeol-dong, Mt. Hambaek)-140605-076 [HNHM]

405. Leonurus japonicus Houtt. 익모초

Taebaek-si(Hwajeon-dong, Mt. Eundae)-140807-014 [HNHM]

406. Leonurus macranthus Maxim. 송장풀

Taebaek-si(Changjuk-dong, Mt. Daedeok)-120920-325 [KB]

$\star 407$. Lycopus lucidus Turcz. 쉽싸리

Taebaek-si(Hwajeon-dong, Mt. Eundae)-140807-160 [HNHM]

408. Meehania urticifolia (Miq.) Makino 벌깨덩굴

Jeongseon-gun(Baekjeon-ri, Mt. Daedeok)-140523-131 [HNHM]

409. Phlomis umbrosa Turcz. 속단

Taebaek-si(Changjuk-dong, Mt. Geumdae)-120704-076 [KB]

410. Prunella vulgaris var. lilacina Nakai 꿀풀
Taebaek-si(Hyeol-dong, Mt. Hambaek)-140704-236 [HNHM]

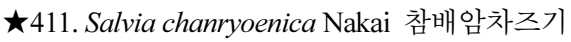

Taebaek-si(Hwajeon-dong, Mt. Eundae)-140807-155 [HNHM]

412. Scutellaria indica L. 골무꽃

Jeongseon-gun(Gohan-ri, Mt. Hambaek)-130828-331 [KB]

$\star$ 413. Scutellaria pekinensis var. transitra (Makino) Hara 산골무꽃

Taebaek-si(Hyeol-dong, Mt. Hambaek)-140704-211 [HNHM]

414. Stachys japonica Miq. 석잠풀

Samcheok-si(Hanso-ri, Mt. Daedeok)-130710-083 [KB]

Solanaceae 가지과

415. Physalis alkekengi var. francheti (Mast.) Hort. 꽈리

Jeongseon-gun(Baekjeon-ri, Mt. Daedeok)-121024-015 [KB]

Scrophulariaceae 현삼과

416. Melampyrum roseum Maxim. 꽃며느리밥풀

Jeongseon-gun(Gohan-ri, Mt. Hambaek)-130711-042 [KB]

$\star$ 417. Melampyrum setaceum var. nakaianum T. Yamaz. 새며느리밥풀 Taebaek-si(Changjuk-dong, Mt. Geumdae)-120815-143 [KB]

$\star 418$. Mimulus nepalensis Benth. 물꽈리아재비

Taebaek-si(Hwajeon-dong, Mt. Eundae)-140807-045 [HNHM]

419. Pedicularis resupinata $\mathrm{L}$. 송이풀

Jeongseon-gun(Gohan-ri, Mt. Hambaek)-130828-232 [KB]

420. Pedicularis resupinata f. albiflora (Nakai) W.T. Lee 흰송이풀

Taebaek-si(Changjuk-dong, Mt. Daedeok)-120920-225 [KB]

\421. Phtheirospermum japonicum (Thunb.) Kanitz 나도송이풀

Taebaek-si(Changjuk-dong, Mt. Daedeok)-120920-021 [KB]

$\star 422$. Scrophularia buergeriana Miq. 현삼

Taebaek-si(Hyeol-dong, Mt. Hambaek)-140704-197 [HNHM]

423. Veronica kiusiana var. glabrifolia (Kitag.) Kitag. 큰산꼬리풀

Taebaek-si(Hyeol-dong, Mt. Hambaek)-140705-250 [HNHM]

$\star 424$. Veronica linariifolia Pall. ex Link 꼬리풀

Jeongseon-gun(Gohan-ri, Mt. Hambaek)-130828-224 [KB]

$\star 425$. Veronica longifolia L. 긴산꼬리풀

Taebaek-si(Hyeol-dong, Mt. Hambaek)-140705-138 [HNHM]

426. Veronica rotunda var. subintegra (Nakai) T. Yamaz. 산꼬리풀

Taebaek-si(Hyeol-dong, Mt. Hambaek)-140704-198 [HNHM]

\section{Orobanchaceae 열당과}

\427. Phacellanthus tubiflorus Siebold \& Zucc. 가지더부살이

Jeongseon-gun(Gohan-ri, Mt. Hambaek)-130711-265 [KB]

Phrymaceae 파리풀과

$\star$ 428. Phryma leptostachya var. asiatica $\mathrm{H}$. Hara 파리풀

Samcheok-si(Hanso-ri, Mt. Daedeok)-130710-090 [KB]

Plantaginaceae 질경이과

429. Plantago asiatica L. 질경이

Taebaek-si(Hwajeon-dong, Mt. Eundae)-140807-069 [HNHM]

430. Plantago polystachya (Makino) Y. Kim 가지질경이

Jeongseon-gun(Baekjeon-ri, Mt. Daedeok)-120814-087 [KB]

Caprifoliaceae 인동과

\431. Lonicera chrysantha Turcz. 각시괴불나무

Jeongseon-gun(Gohan-ri, Mt. Hambaek)-130828-313 [KB]

432. Lonicera coreana Nakai 숫명다래나무

Taebaek-si(Changjuk-dong, Mt. Daedeok)-120419-058 [KB]

$\star$ 433. Lonicera maackii (Rupr.) Maxim. 괴불나무

Taebaek-si(Hyeol-dong, Mt. Hambaek)-140704-160 [HNHM]

434. Lonicera praeflorens Batalin 올괴불나무

Taebaek-si(Changjuk-dong, Mt. Daedeok)-120920-150 [KB]

$\star 435$. Lonicera subsessilis Rehder 청괴불나무

Jeongseon-gun(Gohan-ri, Mt. Hambaek)-130711-188 [KB]

^436. Sambucus sieboldiana var. miquelii (Nakai) Hara 지렁쿠나무

Jeongseon-gun(Gohan-ri, Mt. Hambaek)-140522-065 [HNHM]

437. Viburnum carlesii Hemsl. 분꽃나무 
Taebaek-si(Changjuk-dong, Mt. Daedeok)-120525-330 [KB]

$\star 438$. Viburnum dilatatum Thunb. 가막살나무

Jeongseon-gun(Gohan-ri, Mt. Hambaek)-130711-206 [KB]

439. Viburnum opulus var. calvescens (Rehder) Hara 백당나무

Taebaek-si(Hyeol-dong, Mt. Hambaek)-140704-214 [HNHM]

440. Viburnum wrightii Miq. 산가막살나무

Jeongseon-gun(Gohan-ri, Mt. Hambaek)-120524-026 [KB]

441. Weigela florida (Bunge) A. DC. 붉은병꽃나무

Jeongseon-gun(Baekjeon-ri, Mt. Daedeok)-140523-155 [HNHM]

$\star 442$. Weigela praecox (Lemoine) L.H. Bailey 소영도리나무

Samcheok-si(Hanso-ri, Mt. Daedeok)-130710-046 [KB]

$\star 443$. Weigela subsessilis (Nakai) L.H. Bailey 병꽃나무

Taebaek-si(Hyeol-dong, Mt. Hambaek)-140705-066 [HNHM]

Adoxaceae 연복초과

$\star 444$. Adoxa moschatellina L. 연복초

Jeongseon-gun(Gohan-ri, Mt. Eundae)-140424-082 [HNHM]

Valerianaceae 마타리과

445. Patrinia scabiosaefolia Fisch. ex Trevir. 마타리

Taebaek-si(Hwajeon-dong, Mt. Eundae)-140807-179 [HNHM]

446. Patrinia villosa (Thunb.) Juss. 뚝갈

Samcheok-si(Hanso-ri, Mt. Daedeok)-130710-335 [KB]

447. Valeriana fauriei Briq. 쥐오줌풀

Taebaek-si(Hyeol-dong, Mt. Hambaek)-140605-047 [HNHM]

Campanulaceae 초롱꽃과

448. Adenophora divaricata Franch. \& Sav. 넓은잔대

Taebaek-si(Changjuk-dong, Mt. Geumdae)-120815-046 [KB]

449. Adenophora remotiflora (Siebold \& Zucc.) Miq. 모시대

Taebaek-si(Hwajeon-dong, Mt. Eundae)-140807-043 [HNHM] 450. Adenophora triphylla var. japonica (Regel) H. Hara 잔대 Taebaek-si(Changjuk-dong, Mt. Daedeok)-120920-116 [KB]

451. Campanula glomerata var. dahurica Fisch. 자주꽃방망이 Jeongseon-gun(Gohan-ri, Mt. Hambaek)-140830-001 [HNHM] 452. Campanula punctata Lam. 초롱꽃

Taebaek-si(Hyeol-dong, Mt. Hambaek)-140704-254 [HNHM]

453. Codonopsis lanceolata (Siebold \& Zucc.) Trautv. 더덕

Jeongseon-gun(Baekjeon-ri, Mt. Daedeok)-120814-216 [KB]

Compositae 국화과

$\star 454$. Achillea alpina L. 톱풀

Taebaek-si(Hyeol-dong, Mt. Hambaek)-140705-094 [HNHM]

$\star 455$. Achillea millefolium L. 서양톱풀

Samcheok-si(Hanso-ri, Mt. Daedeok)-130710-292 [KB]

456. Adenocaulon himalaicum Edgew. 멸가치

Jeongseon-gun(Gohan-ri, Mt. Hambaek)-130828-222 [KB]

457. Ainsliaea acerifolia Sch. Bip. 단풍취

Taebaek-si(Hwajeon-dong, Mt. Eundae)-140807-153 [HNHM]

458. Artemisia capillaris Thunb. 사철쑥,

Taebaek-si(Hwajeon-dong, Mt. Eundae)-140807-177 [HNHM]

459. Artemisia gmelini Weber ex Stechm. 더위지기

Taebaek-si(Hwajeon-dong, Mt. Eundae)-140807-184 [HNHM] 460. Artemisia japonica Thunb. 제비쑥

Taebaek-si(Hwajeon-dong, Mt. Eundae)-140807-188 [HNHM] 461. Artemisia keiskeana Miq. 맑은대쑥

Taebaek-si(Hwajeon-dong, Mt. Eundae)-140807-210 [HNHM] 462. Artemisia montana (Nakai) Pamp. 산쑥

Taebaek-si(Hwajeon-dong, Mt. Eundae)-140807-175 [HNHM]

$\star 463$. Artemisia rubripes Nakai 덤불쑥

Taebaek-si(Hwajeon-dong, Mt. Eundae)-140807-174 [HNHM]

464. Artemisia stolonifera (Maxim.) Kom. 넓은잎외잎쑥

Taebaek-si(Hwajeon-dong, Mt. Eundae)-140807-061 [HNHM]

465. Artemisia sylvatica Maxim. 그늘쑥
Taebaek-si(Changjuk-dong, Mt. Daedeok)-120920-089 [KB]

466. Aster ageratoides Turcz. 까실쑥부쟁이

Taebaek-si(Hwajeon-dong, Mt. Eundae)-140807-219 [HNHM]

$\star 467$. Aster incisus Fisch. 가새쑥부쟁이

Jeongseon-gun(Gohan-ri, Mt. Hambaek)-120919-213 [KB]

468. Aster koraiensis Nakai 벌개미취

Jeongseon-gun(Gohan-ri, Mt. Hambaek)-130828-115 [KB]

469. Aster meyendorfii (Regel \& Maack) Voss 개쑥부쟁이

Taebaek-si(Hwajeon-dong, Mt. Eundae)-140807-236 [HNHM]

470. Aster pekinensis (Hance) Chen 가는쑥부쟁이

Jeongseon-gun(Gohan-ri, Mt. Hambaek)-120919-135 [KB]

471. Aster scaber Thunb. 참취

Taebaek-si(Hwajeon-dong, Mt. Eundae)-140807-232 [HNHM]

472. Aster tataricus L. f. 개미취

Jeongseon-gun(Gohan-ri, Mt. Hambaek)-131003-079 [KB]

$\star 473$. Bidens bipinnata $\mathrm{L}$. 도깨비바늘

Jeongseon-gun(Gohan-ri, Mt. Hambaek)-131003-024 [KB]

474. Bidens frondosa $\mathrm{L}$. 미국가막사리

Jeongseon-gun(Gohan-ri, Mt. Hambaek)-120919-259 [KB]

$\star$ 475. Carduus crispus L. 지느러미엉겅퀴

Taebaek-si(Hyeol-dong, Mt. Hambaek)-140605-148 [HNHM]

476. Carpesium abrotanoides L. 담배풀

Jeongseon-gun(Gohan-ri, Mt. Hambaek)-130828-190 [KB]

$\star$ 477. Carpesium divaricatum Siebold \& Zucc. 긴담배풀

Jeongseon-gun(Gohan-ri, Mt. Hambaek)-130828-258 [KB]

$\star$ 478. Carpesium macrocephalum Franch. \& Sav. 여우오줌

Taebaek-si(Hyeol-dong, Mt. Hambaek)-140704-188 [HNHM]

479. Carpesium triste Maxim. 두메담배풀

Jeongseon-gun(Gohan-ri, Mt. Hambaek)-130828-051 [KB]

480. Cirsium japonicum var. maackii (Maxim.) Matsum. 엉겅퀴

Taebaek-si(Hyeol-dong, Mt. Hambaek)-140705-106 [HNHM]

481. Cirsium pendulum Fisch. ex DC. 큰엉겅퀴

Taebaek-si(Hwajeon-dong, Mt. Eundae)-140807-157 [HNHM]

482. Cirsium setidens (Dunn) Nakai 고려엉겅퀴

Jeongseon-gun(Gohan-ri, Mt. Hambaek)-131003-062 [KB]

483. Conyza canadensis (L.) Cronquist 망초,

Jeongseon-gun(Baekjeon-ri, Mt. Daedeok)-120814-136 [HNHM]

484. Crepidiastrum chelidoniifolium (Makino) J.H. Pak \& Kawano 까

치고들빼기

Jeongseon-gun(Gohan-ri, Mt. Hambaek)-130828-163 [KB]

485. Crepidiastrum denticulatum (Houtt.) J.H. Pak \& Kawano 이고들 빼기

Jeongseon-gun(Gohan-ri, Mt. Hambaek)-131003-099 [KB]

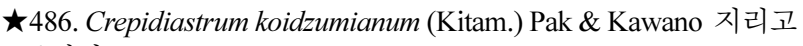
들빼기

Taebaek-si(Hwajeon-dong, Mt. Eundae)-140807-029 [HNHM]

487. Dendranthema boreale (Makino) Ling ex Kitam. 산국

Jeongseon-gun(Baekjeon-ri, Mt. Daedeok)-121024-005 [KB]

^488. Dendranthema sichotense Tzvelev 바위구절초

Jeongseon-gun(Gohan-ri, Mt. Hambaek)-131003-016 [KB]

489. Dendranthema zawadskii var. latilobum (Maxim.) Kitag. 구절초 Jeongseon-gun(Gohan-ri, Mt. Hambaek)-131003-080 [KB]

490. Erigeron annuus (L.) Pers. 개망초

Taebaek-si(Hyeol-dong, Mt. Hambaek)-140705-125 [HNHM]

491. Eupatorium japonicum Thunb. 등골나물

Taebaek-si(Hwajeon-dong, Mt. Eundae)-140807-137 [HNHM]

$\star$ 492. Eupatorium lindleyanum DC. 골등골나물

Jeongseon-gun(Gohan-ri, Mt. Hambaek)-131003-085 [KB]

$\star$ 493. Eupatorium makinoi var. oppositifolium Kawahara \& Yahara 벌등골나물 
Samcheok-si(Hanso-ri, Mt. Daedeok)-130710-141 [HNHM] 494. Galinsoga ciliata (Raf.) S.F. Blake 털별꽃아재비 Taebaek-si(Hyeol-dong, Mt. Hambaek)-140704-226 [HNHM] 495. Hieracium umbellatum L. 조밥나물 Taebaek-si(Hwajeon-dong, Mt. Eundae)-140807-114 [HNHM] 496. Inula britannica var. japonica (Thunb.) Franch. \& Sav. 금불초 Jeongseon-gun(Baekjeon-ri, Mt. Daedeok)-120814-092 [KB] $\star$ 497. Lactuca indica f. indivisa (Makino) Hara 가는잎왕고들빼기 Taebaek-si(Hwajeon-dong, Mt. Eundae)-140807-193 [HNHM] $\star$ 498. Lactuca indica L. 왕고들빼기

Taebaek-si(Hwajeon-dong, Mt. Eundae)-140807-020 [HNHM]

$\star$ 499. Lactuca raddeana Maxim. 산씀바귀

Taebaek-si(Changjuk-dong, Mt. Geumdae)-120815-084 [KB]

500. Lactuca triangulata Maxim. 두메고들빼기

Jeongseon-gun(Gohan-ri, Mt. Hambaek)-130828-144 [KB]

501. Leibnitzia anandria $(\mathrm{L}$.$) Turcz. 솜나물$

Taebaek-si(Changjuk-dong, Mt. Daedeok)-120525-322 [KB]

502. Ligularia fischeri (Ledeb.) Turcz. 곰취

Samcheok-si(Hanso-ri, Mt. Daedeok)-130710-297 [KB]

503. Matricaria matricariodes Porter 족제비쑥

Taebaek-si(Changjuk-dong, Mt. Daedeok)-120525-114 [KB]

504. Parasenecio auriculatus var. kamtschaticus H. Koyama 나래박 쥐나물

Jeongseon-gun(Gohan-ri, Mt. Hambaek)-140522-164 [HNHM]

505. Parasenecio auriculatus var. matsumuranus Nakai 박쥐나물 Jeongseon-gun(Gohan-ri, Mt. Hambaek)-130828-089 [KB]

$\star$ 506. Parasenecio firmus (Kom.) Y.L. Chen 병풍쌈 Jeongseon-gun(Gohan-ri, Mt. Hambaek)-140522-091 [HNHM] 507. Picris hieracioides var. koreana Kitam. 쇠서나물 Taebaek-si(Hwajeon-dong, Mt. Eundae)-140807-172 [HNHM] $\star 508$. Prenanthes tatarinowii Maxim. 개씀배 Jeongseon-gun(Gohan-ri, Mt. Hambaek)-120919-162 [KB] 509. Saussurea gracilis Maxim. 은분취 Jeongseon-gun(Gohan-ri, Mt. Hambaek)-130828-263 [KB]

$\star 510$. Saussurea grandifolia Maxim. 서덜취 Jeongseon-gun(Gohan-ri, Mt. Hambaek)-131003-137 [KB] 511. Saussurea japonica (Thunb.) DC. 큰각시취 Jeongseon-gun(Gohan-ri, Mt. Hambaek)-130828-114 [KB] 512. Saussurea macrolepis (Nakai) Kitam. 각시서덜취 Jeongseon-gun(Gohan-ri, Mt. Hambaek)-130828-046 [KB] 513. Saussurea neoserrata Nakai 산골취 Taebaek-si(Changjuk-dong, Mt. Daedeok)-120920-305 [KB] 514. Saussurea pulchella (Fisch.) Fisch. 각시취 Jeongseon-gun(Gohan-ri, Mt. Hambaek)-130828-165 [KB] $\star 515$. Saussurea tanakae Franch. \& Sav. ex Maxim. 당분취 Taebaek-si(Changjuk-dong, Mt. Geumdae)-120815-018 [KB] 516. Saussurea ussuriensis Maxim. 구와취 Taebaek-si(Changjuk-dong, Mt. Daedeok)-120920-222 [KB] 517. Senecio vulgaris $\mathrm{L}$. 개쑥갓 Jeongseon-gun(Baekjeon-ri, Mt. Daedeok)-140523-141 [HNHM] 518. Serratula coronata var. insularis (Iljin) Kitam. 산비장이 Jeongseon-gun(Gohan-ri, Mt. Hambaek)-131003-147 [KB]

$\star 519$. Solidago serotina Aiton 미국미역취

Jeongseon-gun(Gohan-ri, Mt. Hambaek)-120919-031 [KB] 520. Solidago virgaurea subsp. asiatica Kitam. ex Hara 미역취 Jeongseon-gun(Gohan-ri, Mt. Hambaek)-130828-234 [KB] $\star 521$. Syneilesis palmata (Thunb.) Maxim. 우산나물 Taebaek-si(Hyeol-dong, Mt. Hambaek)-140705-039 [HNHM] 522. Synurus deltoides (Aiton) Nakai 수리취 Jeongseon-gun(Gohan-ri, Mt. Hambaek)-130711-069 [HNHM]
523. Taraxacum officinale Weber 서양민들레

Taebaek-si(Hyeol-dong, Mt. Hambaek)-140605-040 [HNHM]

$\star 524$. Taraxacum ohwianum Kitam. 산민들레

Jeongseon-gun(Baekjeon-ri, Mt. Daedeok)-140523-156 [HNHM]

525. Taraxacum platycarpum Dahlst. 민들레

Jeongseon-gun(Gohan-ri, Mt. Hambaek)-140522-128 [HNHM]

526. Tephroseris flammea (Turcz.) Holub 산솜방망이

Taebaek-si(Changjuk-dong, Mt. Geumdae)-120815-031 [KB]

527. Tephroseris kirilowii (Turcz. ex DC.) Holub 솜방망이

Jeongseon-gun(Gohan-ri, Mt. Hambaek)-120524-394 [KB]

Liliaceae 백합과

528. Allium macrostemon Bunge 산달래

Samcheok-si(Panmun-ri, Mt. Daedeok)-120718-061 [KB]

$\star 529$. Allium monanthum Maxim. 달래

Jeongseon-gun(Gohan-ri, Mt. Hambaek)-140425-185 [HNHM]

$\star 530$. Allium thunbergii G. Don 산부추

Taebaek-si(Changjuk-dong, Mt. Geumdae)-120815-087 [KB]

531. Convallaria keiskei Miq. 은방울꽃

Taebaek-si(Changjuk-dong, Mt. Daedeok)-120525-069 [KB]

532. Disporum smilacinum A. Gray 애기나리

Jeongseon-gun(Gohan-ri, Mt. Hambaek)-130711-243 [KB]

$\star$ 533. Disporum viridescens (Maxim.) Nakai 큰애기나리 Jeongseon-gun(Gohan-ri, Mt. Hambaek)-120524-246 [KH]

534. Erythronium japonicum (Baker) Decne. 얼레지

Jeongseon-gun(Gohan-ri, Mt. Hambaek)-140425-024 [HNHM]

$\star$ 535. Erythronium japonicum f. album T.B. Lee 흰얼레지 Jeongseon-gun(Gohan-ri, Mt. Hambaek)-130501-152 [KB]

$\star 536$. Gagea lutea (L.) Ker-Gawl. 중의무릇

Jeongseon-gun(Gohan-ri, Mt. Eundae)-140424-054 [HNHM]

537. Heloniopsis koreana Fuse, N.S. Lee \& M.N. Tamura 처녀치마 Jeongseon-gun(Gohan-ri, Mt. Hambaek)-130502-113 [KB]

$\star$ 538. Hosta capitata (Koidz.) Nakai 일월비비추

Samcheok-si(Hanso-ri, Mt. Daedeok)-130710-124 [KB]

$\star 539$. Lilium callosum Siebold \& Zucc. 땅나리

Taebaek-si(Changjuk-dong, Mt. Geumdae)-120704-096 [KH]

$\star 540$. Lilium cernuum Kom. 솔나리

Taebaek-si(Changjuk-dong, Mt. Daedeok)-120920-316 [KB]

$\star 541$. Lilium lancifolium Thunb. 참나리

Taebaek-si(Hyeol-dong, Mt. Hambaek)-140705-197 [HNHM]

542. Lilium tsingtauense Gilg 하늘말나리

Taebaek-si(Hyeol-dong, Mt. Hambaek)-140704-119 [HNHM]

$\star$ 543. Lloydia triflora (Ledeb.) Baker 나도개감채

Jeongseon-gun(Gohan-ri, Mt. Eundae)-140424-111 [HNHM]

544. Maianthemum bifolium (L.) F.W. Schmidt 두루미꽃 Jeongseon-gun(Gohan-ri, Mt. Hambaek)-140522-010 [HNHM] 545. Paris verticillata $\mathrm{M}$. Bieb. 삿갓나물 Taebaek-si(Hyeol-dong, Mt. Hambaek)-140605-220 [HNHM] 546. Polygonatum humile Fisher. ex Maxim. 각시둥굴레 Taebaek-si(Changjuk-dong, Mt. Daedeok)-120920-313 [KB] 547. Polygonatum inflatum Kom. 퉁둥굴레 Taebaek-si(Hyeol-dong, Mt. Hambaek)-140605-207 [HNHM] $\star 548$. Polygonatum involucratum (Franch. \& Sav.) Maxim. 용둥굴레 Taebaek-si(Hyeol-dong, Mt. Hambaek)-140605-197 [HNHM] 549. Polygonatum lasianthum Maxim. 죽대 Jeongseon-gun(Gohan-ri, Mt. Hambaek)-120524-302 [KB] 550. Polygonatum odoratum var. pluriflorum (Miq.) Ohwi 둥굴레 Jeongseon-gun(Gohan-ri, Mt. Hambaek)-140522-076 [HNHM]

$\star 551$. Smilacina japonica A. Gray 풀솜대

Jeongseon-gun(Baekjeon-ri, Mt. Daedeok)-140523-005 [HNHM] 552. Smilax nipponica Miq. 선밀나물 
Taebaek-si(Changjuk-dong, Mt. Daedeok)-120525-316 [KB]

$\star$ 553. Smilax sieboldii f. intermis (Nakai) Hara 민청가시덩굴 Taebaek-si(Hyeol-dong, Mt. Hambaek)-140605-037 [HNHM]

554. Streptopus ovalis (Ohwi) F.T. Wang \& Y.C. Tang 금강애기나리

Jeongseon-gun(Gohan-ri, Mt. Hambaek)-140522-170 [HNHM]

555. Trillium kamtschaticum Pall. ex Pursh 연영초

Jeongseon-gun(Gohan-ri, Mt. Hambaek)-140425-175 [HNHM]

$\star 556$. Veratrum maackii Regel 긴잎여로

Jeongseon-gun(Gohan-ri, Mt. Hambaek)-120703-122 [KB]

557. Veratrum maackii var. parviflorum (Maxim.) Hara 파란여로 Jeongseon-gun(Gohan-ri, Mt. Hambaek)-131003-072 [KB]

558. Veratrum oxysepalum Turcz. 박새

Jeongseon-gun(Gohan-ri, Mt. Hambaek)-130624-048 [KB]

Dioscoreaceae 마과

559. Dioscorea nipponica Makino 부채마

Taebaek-si(Hwajeon-dong, Mt. Eundae)-140807-063 [HNHM]

$\star 560$. Dioscorea quinqueloba Thunb. 단풍마

Taebaek-si(Hyeol-dong, Mt. Hambaek)-140704-186 [HNHM]

561. Dioscorea tenuipes Franch. \& Sav. 각시마

Samcheok-si(Hanso-ri, Mt. Daedeok)-130710-162 [KB]

Iridaceae 붓꽃과

$\star 562$. Iris ensata var. spontanea (Makino) Nakai 꽃창포

Jeongseon-gun(Gohan-ri, Mt. Hambaek)-120705-019 [KB]

$\star$ 563. Iris odaesanensis Y.N. Lee 노랑무늬붓꽃

Jeongseon-gun(Gohan-ri, Mt. Hambaek)-120524-224 [KB]

Juncaceae 골풀과

564. Juncus effusus var. decipiens Buchenau 골풀

Taebaek-si(Hyeol-dong, Mt. Hambaek)-140704-054 [HNHM]

$\star 565$. Juncus tenuis Willd. 길골풀

Taebaek-si(Hwajeon-dong, Mt. Eundae)-140807-098 [HNHM]

$\star$ 566. Luzula multiflora Lej. 산꿩의밥

Jeongseon-gun(Gohan-ri, Mt. Hambaek)-140522-148 [HNHM]

Commelinaceae 닭의장풀과

$\star$ 567. Commelina communis L. 닭의장풀

Taebaek-si(Hwajeon-dong, Mt. Eundae)-140807-090 [HNHM]

Gramineae 벼과

$\star$ 568. Agropyron tsukushiense var. transiens (Hack.) Ohwi 개밀

Jeongseon-gun(Gohan-ri, Mt. Hambaek)-090708-70306 [HNHM]

569. Agrostis clavata var. nukabo Ohwi 겨이삭

Taebaek-si(Hyeol-dong, Mt. Hambaek)-140704-201 [HNHM]

$\star 570$. Alopecurus pratensis L. 큰뚝새풀

Jeongseon-gun(Gohan-ri, Mt. Hambaek)-140522-123 [HNHM]

$\star 571$. Anthoxanthum odoratum L. 향기풀

Jeongseon-gun(Gohan-ri, Mt. Hambaek)-120524-003 [KH]

$\star 572$. Arundinella hirta (Thunb.) Koidz. 새

Taebaek-si(Hwajeon-dong, Mt. Eundae)-140807-059 [HNHM]

$\star 573$. Bromus japonicus Thunb. 참새귀리

Taebaek-si(Hyeol-dong, Mt. Hambaek)-140705-200 [HNHM]

$\star 574$. Bromus unioloides Kunth 큰이삭풀

Taebaek-si(Hyeol-dong, Mt. Hambaek)-140705-278 [HNHM]

575. Calamagrostis arundinacea (L.) Roth 실새풀

Jeongseon-gun(Gohan-ri, Mt. Hambaek)-130828-268 [KB]

576. Dactylis glomerata L. 오리새

Samcheok-si(Hanso-ri, Mt. Daedeok)-130710-003 [KB]

$\star 577$. Echinochloa utilis Ohwi \& Yabuno 피

Jeongseon-gun(Baekjeon-ri, Mt. Daedeok)-120814-233 [KB]

578. Festuca ovina L. 김의털

Taebaek-si(Hyeol-dong, Mt. Hambaek)-140705-109 [HNHM]

$\star 579$. Festuca subulata var. japonica Hack. 왕김의털아재비 Jeongseon-gun(Gohan-ri, Mt. Hambaek)-090708-70310 [HNHM]
580. Hierochloe odorata (L.) P. Beauv. 향모

Jeongseon-gun(Gohan-ri, Mt. Hambaek)-120524-227 [KB]

$\star 581$. Lolium perenne L. 호밀풀

Samcheok-si(Panmun-ri, Mt. Daedeok)-120718-220 [KB]

582. Melica nutans $\mathrm{L}$. 왕쌀새

Jeongseon-gun(Gohan-ri, Mt. Eundae)-140424-161 [HNHM]

583. Milium effusum L. 나도겨이삭

Taebaek-si(Hyeol-dong, Mt. Hambaek)-140605-159 [HNHM]

584. Muhlenbergia japonica Steud. 쥐꼬리새

Taebaek-si(Changjuk-dong, Mt. Daedeok)-120920-028 [KB]

$\star$ 585. Panicum dichotomiflorum Michx. 미국개기장

Jeongseon-gun(Gohan-ri, Mt. Hambaek)-130624-062 [KB]

586. Phleum pratense L. 큰조아재비

Jeongseon-gun(Baekjeon-ri, Mt. Daedeok)-140523-117 [HNHM]

$\star 587$. Phragmites japonica Steud. 달뿌리풀

Jeongseon-gun(Gohan-ri, Mt. Hambaek)-120919-170 [KB]

$\star$ 588. Poа аппиа L. 새포아풀

Taebaek-si(Hyeol-dong, Mt. Hambaek)-140704-181 [HNHM]

$\star 589$. Poa hisauchii Honda 구내풀

Jeongseon-gun(Gohan-ri, Mt. Hambaek)-120524-005 [KB]

590. Sasa borealis (Hack.) Makino 조릿대

Taebaek-si(Hwajeon-dong, Mt. Eundae)-140807-143 [HNHM]

591. Setaria viridis (L.) P. Beauv. 강아지풀

Taebaek-si(Changjuk-dong, Mt. Daedeok)-120920-042 [KB]

592. Spodiopogon sibiricus Trin. 큰기름새

Taebaek-si(Hwajeon-dong, Mt. Eundae)-140807-003 [HNHM]

Araceae 천남성과

593. Arisaema amurense Maxim. 둥근잎천남성

Jeongseon-gun(Gohan-ri, Mt. Hambaek)-140522-007 [HNHM]

594. Arisaema amurense f. serratum (Nakai) Kitagausa 천남성

Taebaek-si(Changjuk-dong, Mt. Daedeok)-120525-073 [KB]

595. Arisaema peninsulae Nakai 점박이천남성

Taebaek-si(Changjuk-dong, Mt. Daedeok)-120525-258 [KB]

596. Pinellia ternata (Thunb.) Breitenb. 반하

Taebaek-si(Hyeol-dong, Mt. Hambaek)-140704-151 [HNHM]

Cyperaceae 사초과

597. Carex capillacea Boott 잔솔잎사초

Jeongseon-gun(Gohan-ri, Mt. Hambaek)-120524-064 [KB]

$\star 598$. Carex caryophyllea var. microtricha Kük. 갈색사초

Jeongseon-gun(Gohan-ri, Mt. Hambaek)-140522-022 [HNHM]

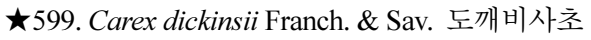

Jeongseon-gun(Gohan-ri, Mt. Hambaek)-130711-028 [KB]

$\star 600$. Carex dispalata Boott 삿갓사초

Jeongseon-gun(Gohan-ri, Mt. Hambaek)-140522-121 [HNHM]

$\star 601$. Carex doniana Spreng. 흰사초

Jeongseon-gun(Gohan-ri, Mt. Eundae)-140424-148 [HNHM]

$\star 602$. Carex echinata Murr 함북사초

Taebaek-si(Hyeol-dong, Mt. Hambaek)-140704-044 [HNHM]

$\star 603$. Carex forficula Franch. \& Sav. 산뚝사초

Jeongseon-gun(Gohan-ri, Mt. Hambaek)-140425-137 [HNHM]

604. Carex humilis var. nana (H. Lév. \& Vaniot) Ohwi 가는잎그늘사초 Jeongseon-gun(Gohan-ri, Mt. Hambaek)-140425-106 [HNHM]

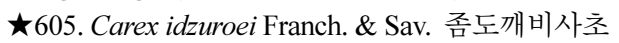

Taebaek-si(Hyeol-dong, Mt. Hambaek)-140605-091 [HNHM]

606. Carex japonica Thunb. 개찌버리사초

Jeongseon-gun(Gohan-ri, Mt. Hambaek)-130624-026 [KB]

607. Carex laevissima Nakai 애괭이사초

Jeongseon-gun(Baekjeon-ri, Mt. Daedeok)-120814-029 [KB]

608. Carex lanceolata Boott 그늘사초

Jeongseon-gun(Gohan-ri, Mt. Hambaek)-140522-089 [HNHM] 
609. Carex leiorhyncha C.A. Mey. 산괭이사초

Samcheok-si(Hanso-ri, Mt. Daedeok)-130710-080 [KB]

610. Carex okamotoi Ohwi 지리대사초

Jeongseon-gun(Baekjeon-ri, Mt. Daedeok)-140523-121 [HNHM]

$\star 611$. Carex onoei Franch. \& Sav. 바늘사초

Jeongseon-gun(Gohan-ri, Mt. Hambaek)-140522-012 [HNHM]

$\star$ 612. Carex pediformis C.A. Mey. 넓은잎그늘사초

Jeongseon-gun(Gohan-ri, Mt. Hambaek)-130501-103 [HNHM]

$\star 613$. Carex planiculmis Kom. 그늘흰사초

Jeongseon-gun(Gohan-ri, Mt. Hambaek)-130711-220 [KB]

$\star$ 614. Carex polyschoena H. Lév. \& Vaniot 가지청사초

Jeongseon-gun(Gohan-ri, Mt. Hambaek)-130411-017 [KB]

$\star 615$. Carex remotiuscula Wahlenb. 층실사초

Taebaek-si(Hyeol-dong, Mt. Hambaek)-140605-114 [HNHM]

616. Carex siderosticta Hance 대사초

Jeongseon-gun(Gohan-ri, Mt. Hambaek)-120524-300 [KB]

Orchidaceae 난초과

617. Cephalanthera longibracteata Blume 은대난초

Samcheok-si(Hanso-ri, Mt. Daedeok)-130710-145 [KB]

618. Liparis krameri Franch. \& Sav. 나나벌이난초

Samcheok-si(Panmun-ri, Mt. Daedeok)-120718-016 [KB]

619. Liparis kumokiri F. Maek. 옥잠난초

Taebaek-si(Hyeol-dong, Mt. Hambaek)-140705-015 [HNHM]

$\star 620$. Oreorchis patens (Lindl.) Lindl. 감자난초

Jeongseon-gun(Baekjeon-ri, Mt. Daedeok)-140523-022 [HNHM]

$\star 621$. Platanthera sachalinensis F. Schmidt 큰제비란

Taebaek-si(Changjuk-dong, Mt. Geumdae)-120704-140 [KB]

$\star 622$. Tulotis ussuriensis (Regel \& Maack) Hara 나도잠자리란

Jeongseon-gun(Gohan-ri, Mt. Hambaek)-120705-124 [KB]

The number behind scientific name means voucher specimen number submitted to National History Museum, Hannam University[HNHM], Korea National Arboretum[KH], National Institute of Biological Resources[KB]; $\star$, Newly found taxa in this investigation. 